\title{
Compressive Blind Image Deconvolution
}

\author{
Bruno Amizic, Member, IEEE, Leonidas Spinoulas, Student Member, IEEE, Rafael Molina, Member, IEEE, \\ and Aggelos K. Katsaggelos, Fellow, IEEE
}

\begin{abstract}
We propose a novel blind image deconvolution (BID) regularization framework for compressive sensing (CS) based imaging systems capturing blurred images. The proposed framework relies on a constrained optimization technique, which is solved by a sequence of unconstrained sub-problems, and allows the incorporation of existing CS reconstruction algorithms in compressive BID problems. As an example, a non-convex $l_{p}$ quasi-norm with $0<p<1$ is employed as a regularization term for the image, while a simultaneous auto-regressive regularization term is selected for the blur. Nevertheless, the proposed approach is very general and it can be easily adapted to other state-ofthe-art BID schemes that utilize different, application specific, image/blur regularization terms. Experimental results, obtained with simulations using blurred synthetic images and real passive millimeter-wave images, show the feasibility of the proposed method and its advantages over existing approaches.
\end{abstract}

Index Terms-Inverse methods, compressive sensing, blind image deconvolution, constrained optimization.

\section{INTRODUCTION}

D IGITAL imaging devices have improved considerably over the years, thus becoming increasingly popular in many different technical areas. Two of their basic requirements are the fulfillment of acquisition and compression. During the acquisition process, the challenge is to capture an image with the highest possible spatial resolution. On the other hand, during compression, the challenge is to store images efficiently and reliably. Often, improved visual quality is synonymous to greater number of imaging sensors and therefore higher sampling rates. In conventional imaging systems the accurate representation of the captured scene is achieved by enforcing the Nyquist sampling theorem. The Nyquist theorem states that the image acquisition sampling rate should be at least twice the scene's highest frequency.

Recently, several imaging devices have been proposed in which acquisition and compression are performed simultaneously, [2]-[4]. They are based on the compressive sensing (CS) theory and require that the signals of interest are sparse or have an approximately sparse representation in a

Manuscript received November 1, 2012; revised March 26, 2013; accepted May 20, 2013. Date of publication June 4, 2013; date of current version August 30, 2013. This work was supported in part by the Department of Energy under Contract DE-NA0000457, the "Ministerio de Ciencia e Innovación" under Contract TIN2010-15137, and the CEI BioTic with the Universidad de Granada. The associate editor coordinating the review of this manuscript and approving it for publication was Prof. Alessandro Foi.

B. Amizic, L. Spinoulas, and A. K. Katsaggelos are with the Department of Electrical Engineering and Computer Science, Northwestern University, Evanston, IL 60208-3118 USA (e-mail: amizic@ northwestern.edu; leonidasspinoulas2015@u.northwestern.edu; aggk@eecs.northwestern.edu).

R. Molina is with the Departamento de Ciencias de la Computación e I.A., Universidad de Granada, Granada 18071, Spain (e-mail: rms@decsai.ugr.es).

Color versions of one or more of the figures in this paper are available online at http://ieeexplore.ieee.org.

Digital Object Identifier 10.1109/TIP.2013.2266100 transformation domain (basis), such as Wavelet or Fourier. Through a CS setting, far fewer measurements, than the desired resolution, are required to accurately reconstruct such signals, [5]-[7]. These measurements, however, are different from the samples that traditional analog-to-digital converters utilize. Instead of simple point evaluations, each measurement is obtained through an inner product of the signal with a different measurement vector. Measurement reduction allows the front ends of imaging devices to become simpler, smaller, and cheaper. This property is desirable for all imaging devices but especially for cumbersome and expensive ones, such as those that capture radiation in the non-visible spectrum (e.g., X-rays, infrared or millimeter wave cameras).

The standard formulation of the CS model is given in matrix-vector form by

$$
\mathbf{y}=\Phi \mathbf{x}+\mathbf{n},
$$

where the $N \times 1$ vector $\mathbf{x}$ and the $M \times 1$ vector $\mathbf{n}$ represent the lexicographically ordered unknown image and the observation noise, respectively. The $M \times N$ matrix $\Phi$ represents the CS measurement matrix while the $M \times 1$ vector $\mathbf{y}$ corresponds to the CS observations. The unknown image, $\mathbf{x}$, is assumed to be of size $m \times n=N$. Note that $M \ll N$.

Extending the CS principles to general purpose acquisition devices is not a straightforward task. Many signals are naturally sparse (e.g., radar pulses) or compressible (e.g., natural images), yet the sparsity assumption for the vector $\mathbf{x}$ is often an oversimplification which does not exploit possible knowledge about the structure of a signal. Although structured sparsity models (e.g., Markov random fields in [8]) have proven efficient in capturing the sparsity structure of specific signals, they cannot be generalized for any type of signal. For example, in [9], [10], it was shown that the spikes present during an electrophysiological recording of a neuron are far better modeled by a stream of impulse responses rather than a series of impulses. Furthermore, the authors in [9], [10] proposed richer sparsity schemes that capture the convolutional structure of pulse streams by defining the following model,

$$
\mathbf{y}=\Phi \mathbf{H x}+\mathbf{n},
$$

where the $N \times N$ matrix $\mathbf{H}$ represents the convolution matrix created from the neuron's impulse response (denoted by the $N \times 1$ vector $\mathbf{h}$ ), and the $N \times 1$ vector $\mathbf{x}$ is assumed to be sparse. The authors in [10] derived the lower bound for the number of measurements needed to accurately reconstruct the sparse vector $\mathbf{x}$, and proposed a reconstruction algorithm which recovers simultaneously the unknown vectors $\mathbf{x}$ and $\mathbf{h}$ for several real-world acquisition systems (e.g., neuronal pulse streams and astronomical imaging). One of the disadvantages 
of the method in [10] is its inability to recover the unknown vectors when pulses overlap.

Neuronal pulse streams fall in the category of filtered sparse processes [11]. However, filtered sparse processes arise in a number of contexts, such as nuclear radiation [12], reflection seismology [13], [14], or communications [11]. The authors in [15] use the same model as in (2) for filtered sparse processes and establish sufficient conditions for exact recovery of both $\mathbf{x}$ and $\mathbf{h}$ when the unknown system $\mathbf{H}$ is auto-regressive of a known order.

Similarly, in [16] the authors proposed the model in (2) to reduce the measurements performed by satellites and aerospace probes of the next generation deep-space imaging systems, hence alleviating the problems of storage, power consumption and transmission without degrading the spatial resolution or the picture quality. In [16], the authors assumed that the acquired images are sparse in a transformed domain and that the $N \times N$ matrix $\mathbf{H}$ represents the blurring matrix created from a known blurring point spread function (PSF) which exists due to the atmosphere, unsuited focal length or relative motion, among other factors. The authors applied curvelet regularization and the Poisson singular integral (PSI) deblurring operator for the purposes of CS deconvolution. Extensions of [16] were presented in [17], where curvelet regularization was combined with total variation (TV) regularization for improved performance, and in [18], where geometric transformations of the imaging system were taken into account while the PSF was estimated through calibration procedures.

The above analysis shows that the model introduced by (2) has numerous applications which are recently being explored. However, we should note an important distinction between the aforementioned techniques. Methods [9], [10], [15] refer to signals that are sparse in the spatial (or time) domain. For such signals, a smooth impulse response $\mathbf{h}$ has the effect of spreading out the signal (e.g., spikes), reducing its sparsity, since the number of nonzero entries increases. On the other hand, methods [16]-[18] deal with images that are sparse in a transformed domain (e.g., frequency). In this case, the effect of convolving the image with a PSF is equivalent to low-pass filtering of the signal which reduces the nonzero coefficients in the transformed domain, sparsifying the signal further and therefore permitting easier reconstruction of its blurred version. However, in this case, additional steps need to be followed to obtain the blur-free version of the signal.

We adopt the second approach, assuming that the signals of interest (images) are compressible and utilize the model presented in (2) to describe the acquisition process of lensbased CS imaging systems. Matrix $\mathbf{H}$ can be viewed as the convolution matrix resulting from the PSF of the lens, limited aperture dimensions, lack of focus, atmospheric turbulences or combinations of the above. For an imaging system, in general, $\mathbf{h}$ cannot always be known a priori and its estimation is essential for the accurate reconstruction of sharp images. In this work, we propose an approach to estimate both the unknown image $\mathbf{x}$ and the blur $\mathbf{h}$ through CS measurements. We provide a BID regularization framework, based on constrained optimization techniques, which allow the use of existing CS reconstruction algorithms in compressive BID problems. To emphasize on the practical applications of the acquisition model, in the experimental section, we propose the reconstruction/restoration of passive millimeter wave (PMMW) images acquired through lens-based CS imaging systems.

This paper is organized as follows. We provide an overview of existing CS reconstruction algorithms and explain the basis for the proposed compressive BID reconstruction algorithm in section II. The proposed CS BID algorithm is analyzed in section III. Finally, supporting experimental results are provided in section IV, and conclusions are drawn in section V.

\section{Compressive Sensing Reconstruction AlgORITHMS}

CS image recovery calls for estimating the sparse or compressible vector $\mathbf{x}$ from the noisy observation $\mathbf{y}$ in (1). It is widely known that this is an ill-conditioned problem, and that simple recovery algorithms, based on least-squares techniques, are unable to accurately recover the unknown sparse vector $\mathbf{x}$. Instead, the most commonly used approach is to solve the following optimization problem,

$$
\underset{\mathbf{x}}{\operatorname{minimize}}\|\mathbf{y}-\Phi \mathbf{x}\|^{2}+\tau\|\mathbf{x}\|_{1},
$$

where $\|\cdot\|$ denotes the Euclidean norm, $\|\cdot\|_{1}$ denotes the $l_{1}$-norm, and $\tau$ is a non-negative regularization parameter. In the last few years, numerous approaches have been proposed to solve this optimization scheme; for example, linear programing methods (e.g., [19]), stochastic approximation methods (e.g., [20]), interior-point methods (e.g., [21]) or Bayesian formulation methods (e.g., [22]), among others. Finding efficient and accurate solutions for the sparse vector $\mathbf{x}$ is still a very active research area.

\section{A. Reconstruction of Compressible Signals}

Many real-world signals are actually not sparse in the acquisition domain and reconstruction methods, based on the model shown in (1) and the optimization problem shown in (3), cannot be applied directly. However, a commonly used assumption is that the signal of interest $\mathbf{x}$ is sparse in the transformed domain (i.e., $\mathbf{x}=\mathbf{W a}$, where the sparse vector a represents the transformed coefficients corresponding to $N$ basis vectors which span the column space of the $N \times N$ matrix $\mathbf{W}$ ). This assumption allows us to utilize state-of-the-art CS algorithms, in their original forms, by solving the following optimization problem,

$$
\underset{\mathbf{a}}{\operatorname{minimize}}\|\mathbf{y}-\Phi \mathbf{W a}\|^{2}+\tau\|\mathbf{a}\|_{1} \text {. }
$$

To complicate things further, many signals of interest are not even sparse in the transformed domain. A commonly used assumption at this point is that such signals are compressible (see [23], [24]). The term compressible refers to signals which can be approximated well by a linear combination of $K(K \ll$ $N$ ) basis vectors from the column space of the matrix W. In addition, the magnitude of their sorted transformed coefficients 

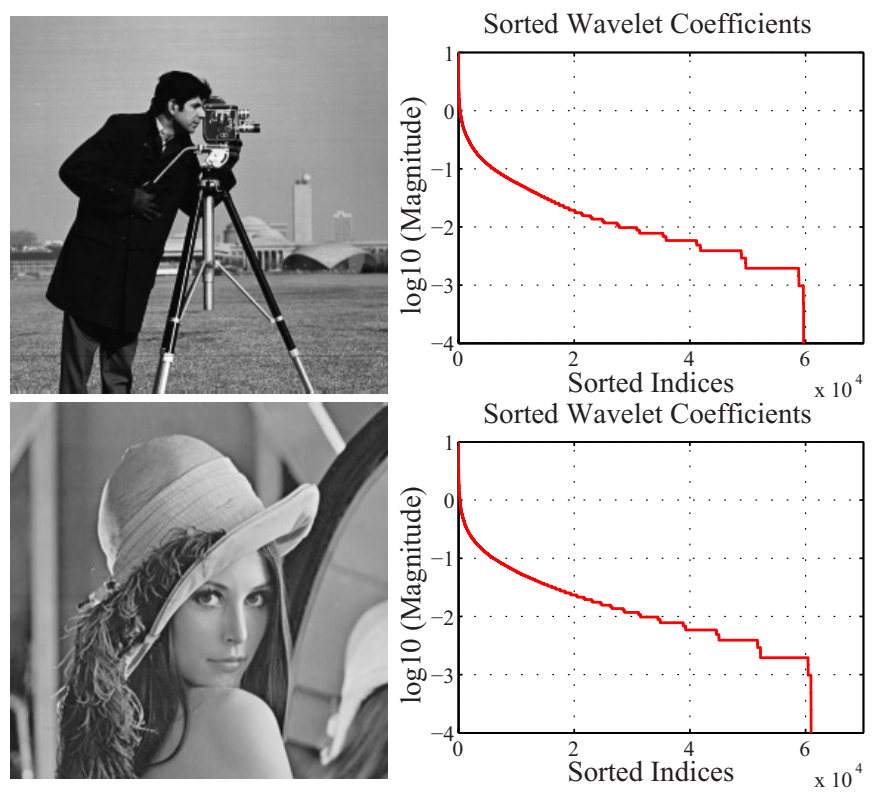

Fig. 1. Examples: $1^{\text {st }}$ column represents the original images; $2^{\text {nd }}$ column represents rapidly decaying magnitudes of the sorted wavelet coefficients, through the Haar wavelet transform, for their respective original images of the $1^{\text {st }}$ column. Note that the image intensities have been normalized.

(denoted by $\mathbf{a}_{s}$ ) must decay rapidly. This rapid decay is often modeled by the power-decay law

$$
\left|\mathbf{a}_{s}(i)\right| \leq \text { Const } \cdot i^{-q}, \quad i=1,2, \ldots, N,
$$

where $q \geq 1$. The magnitude exhibits higher decay rates for larger $q$. Figure 1 demonstrates the rapid magnitude decay of $\mathbf{a}_{s}$ for two standard images that are referenced in many image processing papers.

In [23] it was shown that, if the magnitude of $\mathbf{a}_{s}$ decays as described by the power-decay law in (5), the original signal $\mathbf{x}$ can be well approximated by keeping only the $K$-largest entries (in magnitude) of vector a and zeroing out the remaining ones. Let us denote such approximation of the original signal $\mathbf{x}$ by $\mathbf{x}_{K}$. The authors in [23] also provided an upper bound for the error term for $q \geq 1$ as follows,

$$
\left\|\mathbf{x}-\mathbf{x}_{K}\right\| \leq C_{1} \cdot K^{1 / 2-q},
$$

where $C_{1}$ is a non-negative constant. In Figure 2 we show enlarged image patches of the reconstructions obtained by keeping the $K$-largest entries of vector a. It is observed that even the reconstructions obtained by keeping only $10 \%$ of the largest entries yield visually acceptable results.

\section{B. Reconstruction of Blurred Signals}

As already discussed in section I, blurring is unavoidable in many imaging systems. Here, we analyze some common types of blur, encountered in real-world imaging systems, and show that blurred signals are indeed compressible and usually exhibit faster decay rates for the magnitude of their wavelet coefficients than their respective original versions. Let us first introduce the analytical models for such blurs.

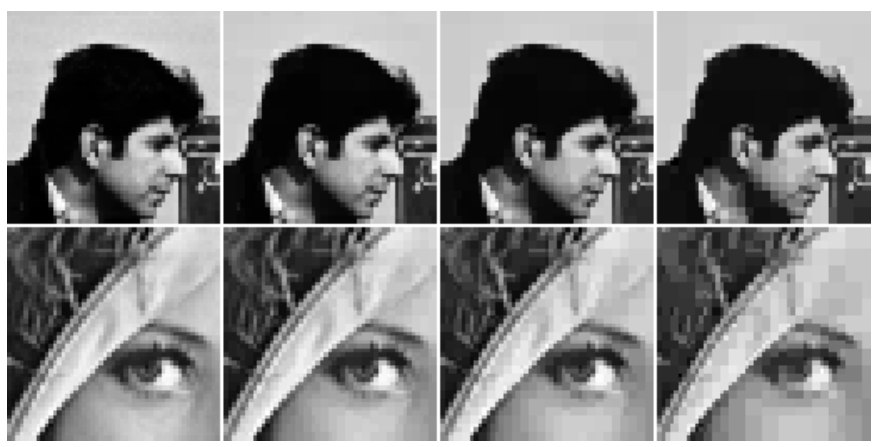

Fig. 2. Examples: $1^{\text {st }}$ column represents enlarged image patches of the original normalized images in Figure $1 ; 2^{\text {nd }}, 3^{r d}$ and $4^{\text {th }}$ columns represent, respectively, the reconstructions obtained by keeping the largest 30\%, 20\% and $10 \%$ of the wavelet coefficients, through the Haar wavelet transform.

1) Motion Blur: This type of blur represents the onedimensional (1D) local averaging of neighboring pixels, a common result of camera panning or fast object motion. For example, the horizontal motion blur can be modeled by

$$
h(\mathbf{u})=\left\{\begin{array}{ll}
\frac{1}{L+1}, & -\frac{L}{2} \leq u_{1} \leq \frac{L}{2} ; \quad u_{2}=0 \\
0, & \text { otherwise }
\end{array},\right.
$$

where $L$ is assumed to be an even integer and $\mathbf{u}=\left(u_{1}, u_{2}\right)$. Motion blur may also occur in two dimensions (2D), e.g., following a random trajectory, as a rotation, etc.

2) Atmospheric Turbulence Blur: This type of blur represents a long term exposure through the atmosphere which is typically modeled by a Gaussian PSF,

$$
h(\mathbf{u})=K e^{-\frac{|\mathbf{u}|^{2}}{2 \sigma^{2}}}
$$

where $K$ is a normalizing constant ensuring that the blur has unit volume, and $\sigma^{2}$ is the variance that determines the severity of the blur.

3) Uniform Out-of-Focus Blur: This type of blur is primarily due to the effects at the camera aperture which depend on various parameters. These parameters include focal length, camera aperture size and shape, distance between camera and observed scene, wavelength of the incoming radiation, and the effects due to diffraction. Accurate knowledge of all these parameters is not frequently available after a picture is taken. When the blur due to poor focusing is large, the following uniform model has been used as an approximation of such PSFs,

$$
h(\mathbf{u})=\left\{\begin{array}{cl}
\frac{1}{\pi r^{2}}, & |\mathbf{u}| \leq r \\
0, & \text { otherwise }
\end{array},\right.
$$

where $r$ denotes radius and controls the severity of the blur.

In Figure 3 we analyze the effect of blurring on the decay rates of the magnitude of the sorted wavelet coefficients for the standard "Cameraman" and "Lena" images, presented in Figure 1. First we degrade the "Cameraman" image with Gaussian blurs of variance 3 and 9. Second, we degrade the "Lena" image with horizontal motion blurs of length 5 and 10 pixels. In both cases, blurring results in a more rapid decay for the magnitude of the sorted wavelet coefficients, 


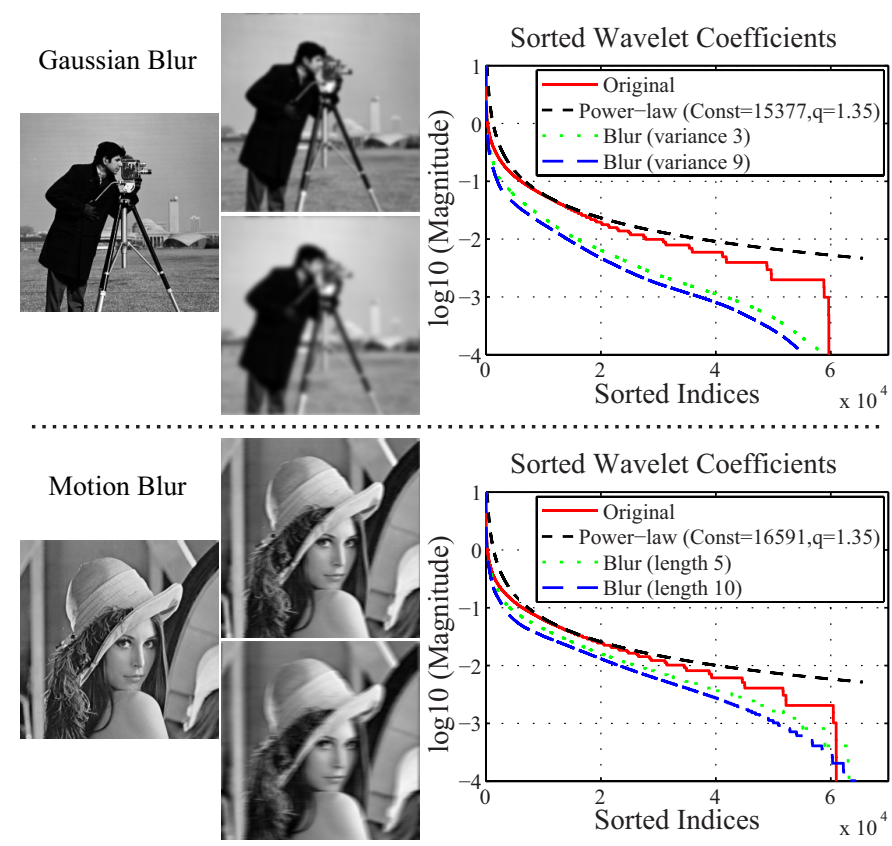

Fig. 3. Upper part: $1^{\text {st }}$ column represents the original normalized image; $2^{\text {nd }}$ column shows the resulting degraded images with a Gaussian blur of variance 3 and 9 , respectively; $3^{\text {rd }}$ column depicts rapidly decaying magnitudes of the sorted wavelet coefficients for the presented images. Lower part: $1^{\text {st }}$ column represents the original normalized image; $2^{\text {nd }}$ column shows the resulting degraded images with a horizontal motion blur of length 5 and 10 pixels, respectively; $3^{\text {rd }}$ column depicts the rapidly decaying coefficients for the presented images. In both graphs we provide the best fitting decaying power-law that bounds the magnitude of the wavelet coefficients for the original image, according to equation (5). For the calculation of the wavelet coefficients, the Haar wavelet transform was used. Values smaller than $10^{-4}$ were pruned away for visualization purposes.

compared to the respective magnitudes of the original images. At the same time, while the severity of the blur increases, the decay rates increase as well. The upper part of Figure 4 presents the equivalent results for the "Shepp-Logan" image degraded with uniform blurs of variance 3 and 9. In the lower part of Figure 4, the same experiment is repeated for an MRI image with Gaussian blurs of variance 1 and 4 . At first glance, the results for the "Shepp-Logan" image appear to contradict the observations of Figure 3. Even though the magnitude of the wavelet coefficients for the blurred images decays faster initially, the rate of decrease soon changes and becomes slower than the corresponding rate for the original image. On the other hand, for the MRI image, the decay rates for the blurred signal follow the observations of Figure 3. Our analysis demonstrates that blurred signals are indeed compressible, obeying the power-law decay of equation (5). Examples for the values of the parameters Const and $q$ of equation (5), when it is fitted to the magnitudes of the wavelet coefficients of the original images, are also presented in the graphs of Figures 3 and 4 . Similar experiments were conducted with a series of images degraded with all three types of blur presented here and converged to the following observation.

For natural images (e.g., "Cameraman", "Lena"), which usually contain a significant amount of edges while exhibiting smooth variations of their image intensities, blurring leads to faster decay rates for the magnitude of their wavelet
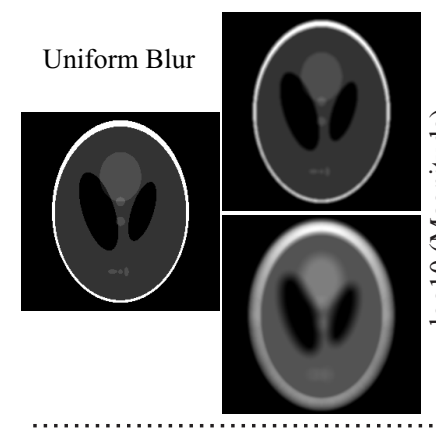

Sorted Wavelet Coefficients
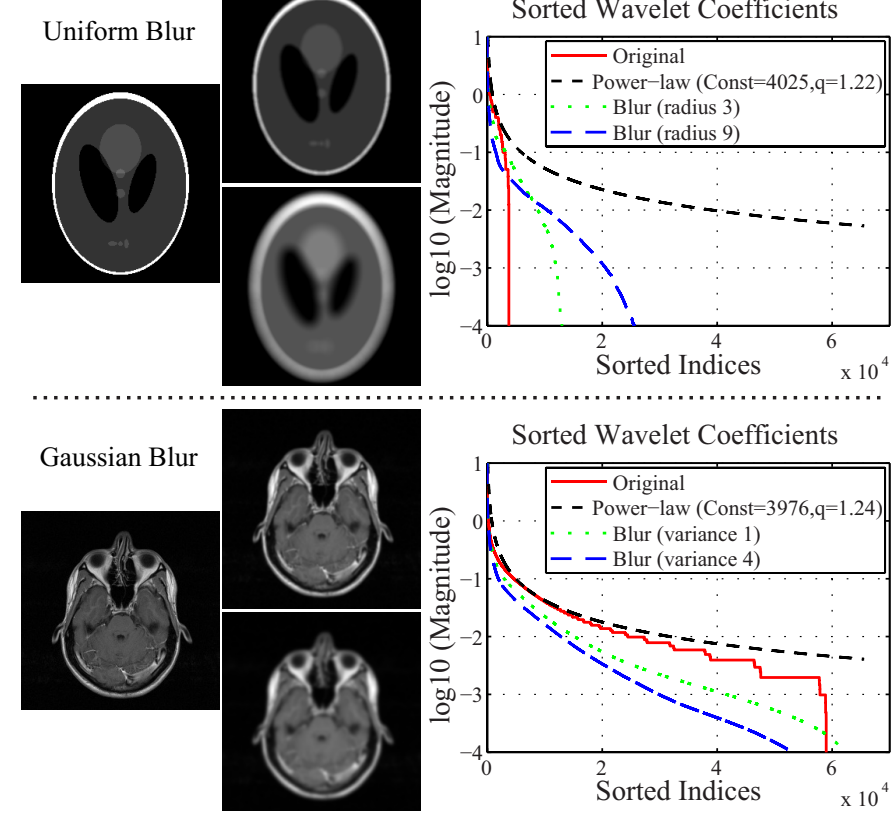

Sorted Wavelet Coefficients

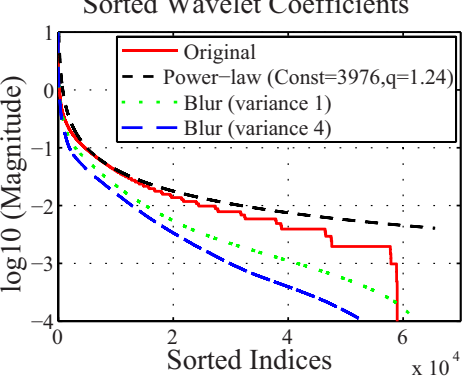

Fig. 4. Upper part: $1^{\text {st }}$ column represents the original normalized image; $2^{\text {nd }}$ column shows the resulting degraded images with a uniform blur of radius 3 and 9 , respectively; $3^{r d}$ column depicts the rapidly decaying magnitudes of the sorted wavelet coefficients for the presented images. Lower part: $1^{\text {st }}$ column represents the original normalized image; $2^{\text {nd }}$ column shows the resulting degraded images with a Gaussian blur of variance 1 and 4 , respectively; $3^{r d}$ column depicts the rapidly decaying coefficients for the presented images. In both graphs we provide the best fitting decaying power-law that bounds the magnitude of the wavelet coefficients for the original image, according to equation (5). For the calculation of the wavelet coefficients, the Haar wavelet transform was used. Values smaller than $10^{-4}$ were pruned away for visualization purposes.

coefficients. Although additional coefficients retain non-zero values, their magnitude is insignificantly small and the blurred signal can be well approximated by less coefficients than the original one, hence making it more compressible and easier to reconstruct. On the contrary, for piece-wise smooth (usually synthetic) images, which contain only few edges with extreme intensity variations (e.g., "Shepp-Logan"), blurring has the opposite effect, since the additional wavelet coefficients in the blurred signal still maintain significant magnitudes containing essential information for accurate signal reconstruction. Nevertheless, piece-wise smooth images whose edges are not as sharp, such as the MRI image, exhibit similar behavior to the natural images. Therefore, fewer measurements would be required to attain a certain reconstruction error, as dictated by equation (6).

\section{Simultaneous Compressive Sensing and Blind Deconvolu- tion}

In this paper, our focus is to establish a robust framework in which existing, state-of-the-art, CS algorithms can be conveniently incorporated for solving the compressed BID problem, as defined in (2), where both image $\mathbf{x}$ and blur $\mathbf{h}$ are unknown. This goal is accomplished exploiting the compressibility of the blurred signals, as explained in section II-B, and the relation $\mathbf{H x}=$ Wa. Therefore, we can now recover the blurred signal 
Hx by solving the typical CS optimization problem

$$
\underset{\mathbf{a}}{\operatorname{minimize}}\|\mathbf{y}-\Phi \mathbf{W a}\|^{2}+\tau\|\mathbf{a}\|_{1} .
$$

The goal of BID algorithms is to recover the unknown image and the unknown blur. Many existing BID approaches are formulated as optimization problems (see [25] for the most recent literature review), where a cost function, with a number of regularization constraint terms, is minimized. Let us denote the solution of (10) by $\hat{\mathbf{a}}$ and the reconstructed blurred signal by $\hat{\mathbf{z}}$ (i.e., $\hat{\mathbf{z}}=\mathbf{W} \hat{\mathbf{a}}$ ). The cost function is chosen as the error function $\|\hat{\mathbf{z}}-\mathbf{H x}\|^{2}$ which ensures fidelity to the data. It is well-known that the BID problem is ill-posed, [25], and that regularization terms for the image and the blur are needed. The regularization terms are used to impose additional constraints on the unknowns. The effect of the regularization terms is controlled by regularization parameters, that basically represent the trade-off between fidelity to the data and the desirable properties (e.g., piece-wise smoothness) of the solution. A general form of the functional to be minimized, using BID techniques, is

$$
\underset{\mathbf{x}, \mathbf{h}}{\operatorname{minimize}} \frac{\beta}{2}\|\hat{\mathbf{z}}-\mathbf{H x}\|^{2}+\alpha R_{1}(\mathbf{x})+\gamma R_{2}(\mathbf{h}),
$$

where $\beta, \alpha, \gamma$ are non-negative regularization parameters, and $R_{1}(\cdot)$ and $R_{2}(\cdot)$ are some regularization functionals. Note that, we intentionally did not specify both the regularization terms for the image and blur to keep the framework as general as possible. In practice, the regularization terms should be chosen based on some prior assumptions about the unknowns. For example, if an image is expected to be piece-wise smooth, introducing the total variation (TV) regularization for the image is a popular choice (see [26]).

Based on the above analysis, a typical way to solve the CS BID problem would be to follow a sequential approach. That is, one first solves the CS reconstruction problem, using (10), to obtain the blurred signal $\hat{\mathbf{z}}$ and then performs BID, solving the regularized minimization problem (11). However, combining both equations into a simultaneous optimization problem leads to improved performance as we will show in section IV.

Therefore, let us propose the constrained optimization problem which allows us to link the solutions of (10) and (11), and recover, simultaneously, the transformed coefficients a of the blurred image, the unknown image $\mathbf{x}$, and the unknown blur $\mathbf{h}$,

$$
\begin{aligned}
& \underset{\mathbf{x}, \mathbf{h}, \mathbf{a}}{\operatorname{minimize}} \frac{\beta}{2}\|\mathbf{y}-\Phi \mathbf{W a}\|^{2}+\tau\|\mathbf{a}\|_{1}+\alpha R_{1}(\mathbf{x})+\gamma R_{2}(\mathbf{h}) \\
& \text { subject to } \mathbf{H} \mathbf{x}=\mathbf{W a} .
\end{aligned}
$$

The advantage of (12) compared to the sequential CS BID approach is the ability to impose an additional structural constraint on the transformed coefficients a. We accomplish this through the relation $\mathbf{H x}=\mathbf{W} \mathbf{a}$ and by exploiting the convolutional inter-dependencies between the image $\mathbf{x}$ and the blur $\mathbf{h}$ when solving for the transformed coefficients $\mathbf{a}$.

\section{Regularization BASED APPROACH}

Let us now propose an algorithm that finds a solution of the constrained optimization problem (12) by solving a series of unconstrained sub-problems. In this work we adopted the quadratic penalty method (see section 17.1 in [27] for more details). This method suggests the formation of a quadratic functional $Q(\mathbf{a}, \mathbf{h}, \mathbf{x} ; \eta)$ by adding a quadratic penalty term to the original objective function in (12), such that,

$$
\begin{aligned}
Q(\mathbf{a}, \mathbf{h}, \mathbf{x} ; \eta)= & \frac{\beta}{2}\|\mathbf{y}-\Phi \mathbf{W a}\|^{2}+\frac{\eta}{2}\|\mathbf{W a}-\mathbf{H} \mathbf{x}\|^{2}+ \\
& +\tau\|\mathbf{a}\|_{1}+\alpha R_{1}(\mathbf{x})+\gamma R_{2}(\mathbf{h}),
\end{aligned}
$$

where the non-negative parameter $\eta$ is the penalty parameter.

Now, with the addition of the quadratic penalty term to the original objective, we replace the constrained optimization problem defined in (12) by a series of unconstrained optimization sub-problems, of the form,

$$
\underset{\mathbf{x}, \mathbf{h}, \mathbf{a}}{\operatorname{minimize}} Q(\mathbf{a}, \mathbf{h}, \mathbf{x} ; \eta) .
$$

The solution of (14) coincides with the solution of (12) when the non-negative parameter $\eta$ approaches $\infty$ (see section 17.1 in [27] for more details). Some other popular approaches, that could be employed here, are non-smooth exact penalty methods and the method of multipliers or augmented Lagrangian method (see sections 17.2 and 17.3 in [27]). The augmented Lagrangian method is known to reduce the possibility of illconditioning by introducing explicit Lagrangian multipliers into the function to be minimized. However, we did not (experimentally) observe these ill-conditioning effects of the quadratic penalty method, after performing an extensive set of experiments with different blurs and noise levels.

We solve the aforementioned problem by alternating minimization (AM) solving for one unknown while fixing the remaining ones. Clearly, the AM approach sets an optimization framework, in which one can introduce existing state-of-theart CS reconstruction algorithms when solving for the sparse vector a. The update strategy of the AM framework results in the following sequence of unconstrained sub-problems,

$$
\begin{aligned}
\mathbf{x}^{k+1} & =\underset{\mathbf{x}}{\arg \min } Q\left(\mathbf{a}^{k}, \mathbf{h}^{k}, \mathbf{x} ; \eta^{k}\right), \\
\mathbf{h}^{k+1} & =\underset{\mathbf{h}}{\arg \min } Q\left(\mathbf{a}^{k}, \mathbf{h}, \mathbf{x}^{k+1} ; \eta^{k}\right), \\
\mathbf{a}^{k+1} & =\underset{\mathbf{a}}{\arg \min } Q\left(\mathbf{a}, \mathbf{h}^{k+1}, \mathbf{x}^{k+1} ; \eta^{k}\right), \\
\eta^{k+1} & =\theta \eta^{k},
\end{aligned}
$$

where $k$ is the iteration number, and $\theta>1$.

Note that various algorithms can, in fact, be used to calculate the unknown vectors (e.g., steepest descent, conjugate gradient, direct inversion in the discrete Fourier domain, etc.).

\section{A. Implementation Details}

First, let us specify the regularization terms utilized for the image and blur. Their selection is application specific and should be done based on some prior assumptions and expectations. In this work, similarly to [28] and [29], we utilize a non-convex $l_{p}$ quasi norm with $0<p<1$ for the 
image, since the derivatives of blurry images are expected to be sparse. The distributions of the image derivatives often have heavier tails that are better modeled with the non-convex $l_{p}$ quasi norm prior compared to the convex priors modeled with $p=1,2$. We employ a variant of the $l_{p}$ quasi-norm as a regularization term for the image,

$$
R_{1}(\mathbf{x})=\left[\sum_{d \in \mathrm{D}} 2^{1-o(d)} \sum_{i}\left|\Delta_{i}^{d}(\mathbf{x})\right|^{p}\right],
$$

where $o(d) \in\{1,2\}$ denotes the order of the difference operator $\Delta_{i}^{d}(\mathbf{x}), 0<p<1$, and $d \in \mathrm{D}=\{h, v, h h, v v, h v\}$. $\Delta_{i}^{h}(\mathbf{x})$ and $\Delta_{i}^{v}(\mathbf{x})$ correspond, respectively, to the horizontal and vertical first order differences, at pixel $i$, that is, $\Delta_{i}^{h}(\mathbf{x})=$ $u_{i}-u_{l(i)}$ and $\Delta_{i}^{v}(\mathbf{x})=u_{i}-u_{a(i)}$, where $l(i)$ and $a(i)$ denote the nearest neighbors of $i$, to the left and above, respectively. The operators $\Delta_{i}^{h h}(\mathbf{x}), \Delta_{i}^{v v}(\mathbf{x}), \Delta_{i}^{h v}(\mathbf{x})$ correspond, respectively, to horizontal, vertical and horizontal-vertical second order differences, at pixel $i$.

For the blur, we utilize a simultaneous auto-regressive (SAR) regularization, that is,

$$
R_{2}(\mathbf{h})=\|\mathbf{C h}\|^{2},
$$

where $\mathbf{C}$ is the discrete Laplacian operator. The SAR regularization (see [30] for a Bayesian interpretation) imposes smoothness on the blur estimates and it is typically applied as a model for blurs that are expected to be smooth, as is the case of atmospheric turbulence. Our motivation for choosing a smooth prior for the blur is to blindly restore images acquired by the PMMW imager from ANL, [31], for which we are finding that the PSF of the imaging system is smooth. Furthermore, additional studies on PMMW imaging systems (e.g., [32]) have shown that their PSFs exhibit smoothness. Nonetheless, the developed theory can handle non-smooth blurs by appropriately modifying the blur model.

At the same time, as the minimization of the non-convex functional in (14) is not straightforward, we employ the majorization-minimization approach [33] to bound the nonconvex image regularizer $R_{1}(\mathbf{x})$ by the functional $\mathrm{M}_{1}(\mathbf{x}, \mathbf{V})$, that is,

$$
R_{1}(\mathbf{x}) \leq \mathrm{M}_{1}(\mathbf{x}, \mathbf{V}),
$$

where $M_{1}(\mathbf{x}, \mathbf{V})$ is defined as

$$
\mathrm{M}_{1}(\mathbf{x}, \mathbf{V})=\frac{p}{2} \sum_{d \in \mathrm{D}} 2^{1-o(d)} \sum_{i} \frac{\left[\Delta_{i}^{d}(\mathbf{x})\right]^{2}+\frac{2-p}{p} v_{d, i}}{v_{d, i}^{1-p / 2}},
$$

and $\mathbf{V}$ is a matrix with elements $v_{d, i}>0$. Detailed derivation of this bound is presented in [34].

Finally, we present our BID algorithm for compressive sampling. In algorithm 1, we follow a general framework for solving a constrained optimization problem by a series of unconstrained ones (see Chapter 17 in [27] for more details).

Algorithm. Given $\alpha, \beta, \gamma, \tau, \eta^{1}, \mathbf{a}^{1}$, and $\mathbf{V}^{1,1}$, where the rows of $\mathbf{V}^{k, l}$ are denoted by $\mathbf{v}_{d}^{k, l} \in\left(R^{+}\right)^{N}$, with $d \in$ $\{h, v, h h, v v, h v\}$, and an initial estimate of the blur $\mathbf{h}^{1,1}$. For $k=1,2, \ldots$ until a stopping criterion is met:

1) For $l=1,2, \ldots$ until a stopping criterion is met:

\section{1.a) Calculate}

$$
\begin{aligned}
\mathbf{x}^{k, l}=\left[\eta^{k}\left(\mathbf{H}^{k, l}\right)^{t}\left(\mathbf{H}^{k, l}\right)\right. & \left.+\alpha p \sum_{d} 2^{1-o(d)}\left(\boldsymbol{\Delta}^{d}\right)^{t} \mathrm{~B}_{d}^{k, l}\left(\boldsymbol{\Delta}^{d}\right)\right]^{-1} \\
& \times \eta^{k}\left(\mathbf{H}^{k, l}\right)^{t} \mathbf{W a}^{k}
\end{aligned}
$$

where $\mathrm{B}_{d}^{k, l}$ is a diagonal matrix with entries $\mathrm{B}_{d}^{k, l}(i, i)=\left(v_{d, i}^{k, l}\right)^{p / 2-1}$ and $\boldsymbol{\Delta}^{d}$ is the convolution matrix of the difference operator $\Delta_{i}^{d}(\cdot)$.

\section{1.b) Calculate}

$$
\mathbf{h}^{k, l+1}=\left[\eta^{k}\left(\mathbf{X}^{k, l}\right)^{t}\left(\mathbf{X}^{k, l}\right)+\gamma \mathbf{C}^{t} \mathbf{C}\right]^{-1} \times \eta^{k}\left(\mathbf{X}^{k, l}\right)^{t} \mathbf{W a}^{k}(24)
$$

where $\mathbf{X}^{k, l}$ is the convolution matrix of the image $\mathbf{x}^{k, l}$.

1.c) For each $d \in\{h, v, h h, v v, h v\}$ calculate

$$
v_{d, i}^{k, l+1}=\left[\Delta_{i}^{d}\left(\mathbf{x}^{k, l}\right)\right]^{2} .
$$

2) $\operatorname{Set} \mathbf{x}^{k}=\mathbf{x}^{k, l}, \mathbf{h}^{k+1,1}=\mathbf{h}^{k, l+1}, \mathbf{H}^{k}=\mathbf{H}^{k, l+1}$ and $v_{d, i}^{k+1,1}=$ $v_{d, i}^{k, l+1}$ for each $d \in\{h, v, h h, v v, h v\}$.

3) Find

$$
\begin{aligned}
\mathbf{a}^{k+1}= & \arg \min \\
\mathbf{a} & \frac{\beta}{2}\|\mathbf{y}-\Phi \mathbf{W a}\|^{2}+ \\
& +\frac{\eta^{k}}{2}\left\|\mathbf{W a}-\mathbf{H}^{k} \mathbf{x}^{k}\right\|^{2}+\tau\|\mathbf{a}\|_{1} .
\end{aligned}
$$

4) $\mathrm{Set}$

$$
\eta^{k+1}=\theta \eta^{k}, \quad \theta>1 .
$$

Note that, since (26) can be re-written as

$$
\mathbf{a}^{k+1}=\arg \min _{\mathbf{a}}\left\|\mathbf{y}^{\prime}-\Phi^{\prime} \mathbf{W a}\right\|^{2}+\tau\|\mathbf{a}\|_{1},
$$

where $\mathbf{y}^{\prime}=\left[\begin{array}{c}\sqrt{\frac{\beta}{2}} \mathbf{y} \\ \sqrt{\frac{\eta^{k}}{2}} \mathbf{H}^{k} \mathbf{x}^{k}\end{array}\right]$ and $\Phi^{\prime}=\left[\begin{array}{c}\sqrt{\frac{\beta}{2}} \Phi \\ \sqrt{\frac{\eta^{k}}{2}} \mathbf{I}\end{array}\right]$, then when solving for the sparse vector a, existing CS reconstruction algorithms (e.g., [21]) can be employed. Finally, as we have already mentioned, different image and blur regularizers can be used in our framework. This makes the proposed approach a versatile model to solve compressive BID problems.

\section{EXPERIMENTAL RESULTS}

In this section we conduct a series of experiments to support our CS BID approach. In the first part, we perform synthetic experiments and compare our algorithm with existing methods while in the second part, we provide a brief description of passive millimeter wave (PMMW) imaging systems and we test our algorithm on real PMMW images.

For comparison purposes we use a set of state-of-the art algorithms, namely $l 1-l s$ [21], GPSR [35], NESTA [36], YALL1 [37], and CoSaMP [38], whose codes are available online. These algorithms can solve for the signal $\mathbf{x}$ when the CS observation is given by the model in (2) and the convolution matrix $\mathbf{H}$, or equivalently the blur $\mathbf{h}$, is known (non-blind case). Let us denote the vector of sparse coefficients of the original signal in a transformed domain $\mathbf{W}$ by $\mathbf{s}$, such that the original signal is given by $\mathbf{x}=$ Ws.

Algorithms $l 1-l s$ and GPSR solve the problem

$$
\underset{\mathbf{s}}{\operatorname{minimize}}\|\mathbf{y}-\Phi \mathbf{H W s}\|^{2}+\tau\|\mathbf{s}\|_{1} .
$$




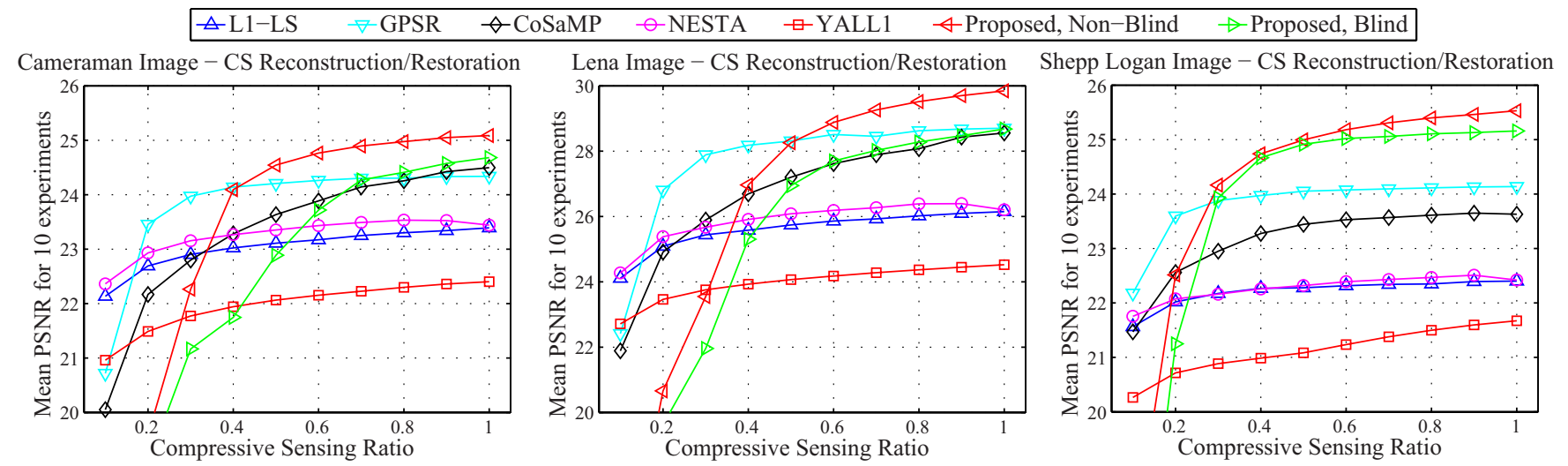

Fig. 5. Average performance comparison of the non-blind and blind versions of the proposed approach with a series of state-of-the-art algorithms, namely, l1-ls [21], GPSR [35], NESTA [36], YALL1 [37], and CoSaMP [38] that solve the non-blind reconstruction/restoration problem. All images are degraded with a Gaussian PSF of variance 5 and Gaussian noise is added to the CS measurements so that SNR $=40 \mathrm{~dB}$. Values smaller than $20 \mathrm{~dB}$ were pruned away for visualization purposes.

Algorithms NESTA and YALL1 solve the analysis-based problem,

$$
\text { minimize }\left\|\mathbf{W}^{-1} \mathbf{x}\right\|_{1} \text { subject to }\|\mathbf{y}-\Phi \mathbf{H} \mathbf{x}\|<\epsilon,
$$

which is equivalent to the problem in (29) when $\mathbf{W}$ corresponds to a basis (e.g., Wavelet). Finally, CoSaMP is based on Orthogonal Matching Pursuit (OMP) schemes and attempts to find an approximation of the signal by restricting its support to a user specified size of $K$.

\section{A. Synthetic Experiments and Discussion}

For the synthetic experiments we use the "Cameraman", "Lena", and "Shepp-Logan" images of size $256 \times 256$ pixels. All images are normalized, degraded with a Gaussian PSF and Gaussian noise is added to the CS measurements. For efficient implementation (due to the large size of the images) we use circulant Gaussian measurement matrices $(\Phi)$. In a detailed comparative study on various CS measurement matrices, [39], circulant and toeplitz Gaussian and Bernoulli measurement matrices have proven to perform equally well to full random Gaussian or Bernoulli matrices. As a performance metric, we use the peak signal to noise ratio (PSNR) which is defined as

$$
\mathrm{PSNR}=10 \log _{10} \frac{N L^{2}}{\|\mathbf{x}-\hat{\mathbf{x}}\|^{2}},
$$

where $\mathbf{x}$ and $\hat{\mathbf{x}}$ are the original and estimated images, respectively, and the constant $L$ represents the maximum possible intensity value in image $\mathbf{x}$. Furthermore, for all experiments, we use the 3-level Haar wavelet transform as our sparsifying basis W. Additional wavelet transforms from the "Daubechies" family were tested providing similar relative performance.

We perform two series of experiments for each image: nonblind reconstructions/restorations when the blur $\mathbf{h}$ is assumed to be known, and blind reconstructions/restorations when the blur $\mathbf{h}$ is unknown and is being estimated.

For the non-blind experiments, the parameters in all the aforementioned algorithms were optimized, based on the suggestions of the authors as well as testing a large set of different values and the best obtained results are presented here. To achieve maximum performance, algorithms NESTA and YALL1 were additionally fed with the real noise variance as an indicator for the appropriate selection of the parameter $\epsilon$, whereas, for the CoSaMP algorithm, we provided an estimate of the original signal sparsity in the wavelet domain by counting the sparse coefficients that have magnitude greater than $10^{-2}$. In our approach we, of course, skip step 1.b) of the proposed algorithm in section III-A, since the blur is assumed to be known. The parameters are set to $\left\{\alpha, \beta, \gamma, \tau, \eta^{l}\right\}=$ $\left\{1,1 / \sigma^{2}, 5 e 5,0.125,1042\right\}$ for the "Cameraman" and "Lena" images and $\left\{\alpha, \beta, \gamma, \tau, \eta^{l}\right\}=\left\{1,1 / \sigma^{2}, 25 e 5,125, \beta / 2^{7}\right\}$ for the "Shepp-Logan" image, where $\sigma^{2}$ denotes the noise variance. The rest of the algorithmic parameters, $p$ and $\theta$ are set to 0.8 and 1.3, respectively, for all images. The number of iterations for the inner loop of the proposed algorithm is set to 2 (in the quadratic penalty method, see [27], it is suggested to calculate an approximate solution for the unknowns at each iteration $k$ ). The algorithm is terminated when the convergence criterion $\left\|\mathbf{x}^{k}-\mathbf{x}^{k-1}\right\| /\left\|\mathbf{x}^{k-1}\right\|<10^{-3}$ is satisfied. For the blind experiments, the parameters of our algorithm stay unaltered but we change the convergence criterion to $\left\|\mathbf{x}^{k}-\mathbf{x}^{k-1}\right\| /\left\|\mathbf{x}^{k-1}\right\|<10^{-2}$. Finally, the initial blur estimate $\mathbf{h}^{1,1}$ is set to a Gaussian PSF of variance 2 .

We conduct experiments for compressive ratios 0.1 to 1 with step 0.1 for all algorithms. 10 experiments are conducted per algorithm for each compressive ratio and the average performance is reported. We test each algorithm under different degradations and noise realizations. Specifically, we degrade each of the tested images with Gaussian PSFs of variance 3, 5 and 9 and add Gaussian noise to the CS measurements so that the signal to noise ratio (SNR) becomes $30 \mathrm{~dB}$ or 40 $\mathrm{dB}$. The results for all conducted experiments are summarized in Table I (on page 4003) where the step of CS ratios is 0.2 due to space limitations. Entries of the table in bold denote the highest average PSNR for each tested set of: image, blur variance, SNR and CS ratio. Additionally, a graphical performance comparison is presented in Figure 5 for all tested images, when they are degraded with a Gaussian PSF of 


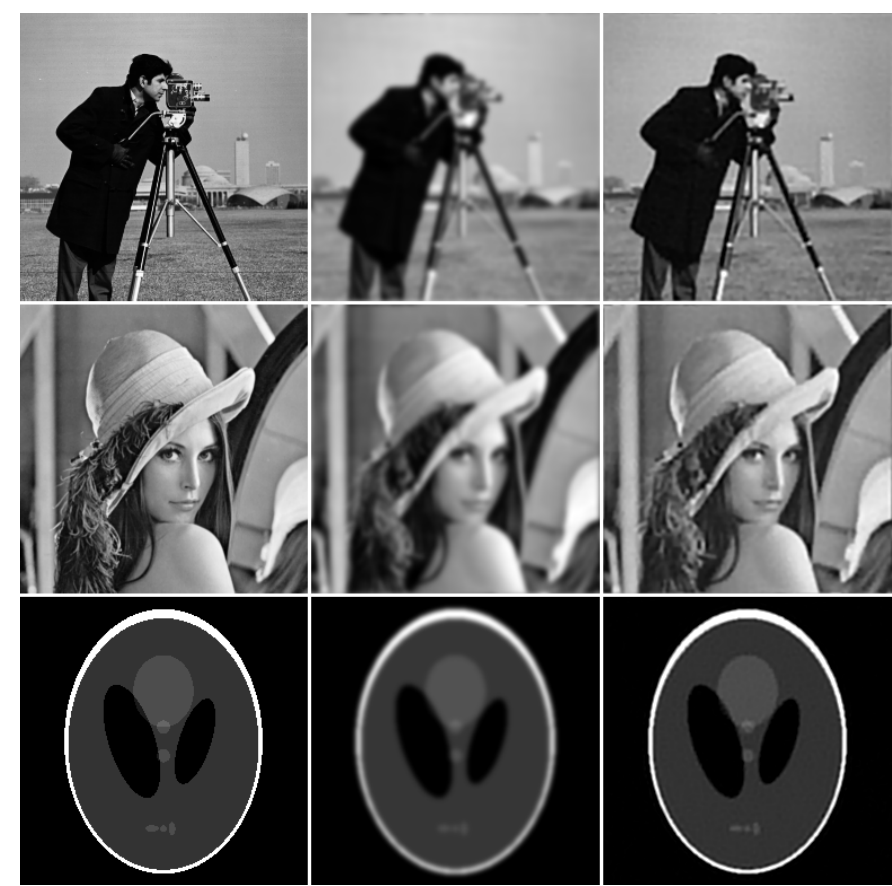

Fig. 6. $1^{\text {st }}$ column represents the original images; $2^{\text {nd }}$ column depicts the images of the $1^{s t}$ column, degraded with a Gaussian PSF of variance $5 ; 3^{\text {rd }}$ column shows the non-blind reconstruction/restoration for SNR $=40 \mathrm{~dB}$ and $100 \%$ of CS measurements, using the proposed algorithm; that is the best image quality one can obtain using the proposed approach.

variance 5 and SNR $=40 \mathrm{~dB}$. Both Table I and Figure 5 document that, even though the non-blind version of our approach exhibits low PSNRs for compressive ratios smaller than 0.3 it soon outperforms most compared algorithms for greater CS ratios. However, note that image quality for ratios $0.1-0.2$ is poor for all the tested approaches and the most meaningful results appear when more CS measurements are acquired. The algorithms that have competitive performance to our approach are GPSR and, CoSaMP only for SNR $=40 \mathrm{~dB}$. Note, however, that the presented results were obtained by setting the convergence tolerance of GPSR to $10^{-5}$ (much lower than our approach). Additionally, CoSaMP requires and was provided with an estimate of the image sparsity which is not usually known a priori. For the blind reconstructions/restorations, our algorithm performs reasonably well, achieving PSNRs close to the non-blind case, especially for the "Shepp-Logan" image. Furthermore, the blind results outperform the non-blind results of some of the compared algorithms for CS ratios over 0.3.

Figures 6 and 7 depict the tested images, their blurry degraded versions with a Gaussian PSF of variance 5, and a series of non-blind and blind reconstructions/restorations for various CS ratios when SNR $=40 \mathrm{~dB}$. The presented images correspond to the highest achieved PSNR out of the 10 tested cases for each CS ratio. It is apparent that images of high visual quality are obtained following the proposed framework. Additionally, in Figure 8, we provide the estimated PSFs for the images presented in Figure 7 together with the real Gaussian PSF of variance 5 used to degrade the original images (in gray background). One can observe that the accuracy of the PSF estimation reduces as compressive

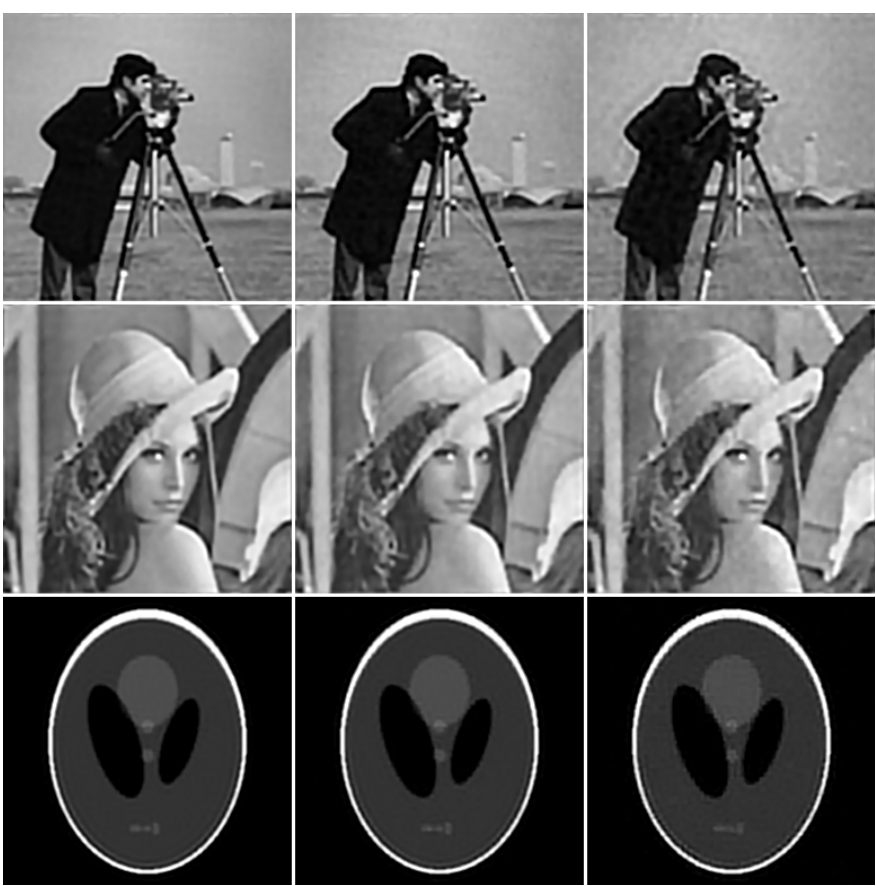

Fig. 7. Blind reconstruction/restoration results, using the proposed algorithm, for the images in Figure 6 (Gaussian PSF of variance 5 and SNR $=40 \mathrm{~dB}$ ) and for compressive ratios $0.8,0.6$, and 0.4 from left to right, respectively. All presented images correspond to the maximum achieved PSNR from the 10 conducted experiments for each compressive ratio.
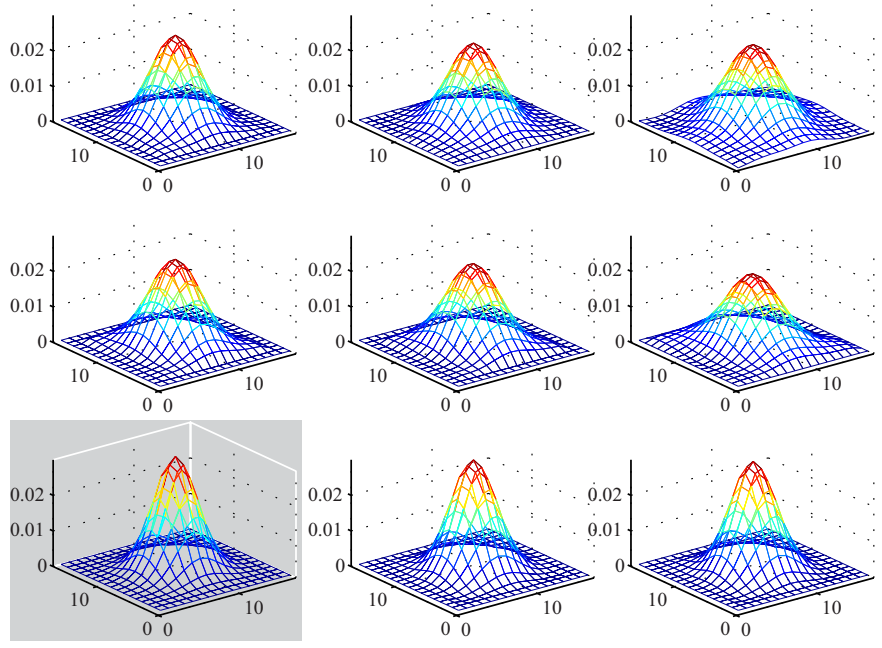

Fig. 8. Estimated PSFs $(17 \times 17$ central part $)$ for the blind reconstruction/restoration results presented in Figure 7 (Gaussian PSF of variance 5 and $\mathrm{SNR}=40 \mathrm{~dB}$ ). $1^{\text {st }}$ row corresponds to the "Cameraman" image; $2^{\text {nd }}$ row corresponds to the "Lena" image; $3^{\text {rd }}$ row corresponds to the "Shepp-Logan" image for compressive ratios $0.8,0.6$ and 0.4 from left to right, respectively. Note that the bottom-left graph with the gray background corresponds to the original Gaussian PSF of variance 5, with which the original images were degraded.

ratio decreases, resulting in lower image quality and some ringing artifacts (e.g., "Cameraman" image for CS ratio 0.4 at the upper-right corner of Figure 7). However, for the "SheppLogan" image, PSF estimation is almost exact for a broad range of CS ratios.

Now, we support our claim, in section II-C, that the proposed simultaneous CS BID approach is superior to the 

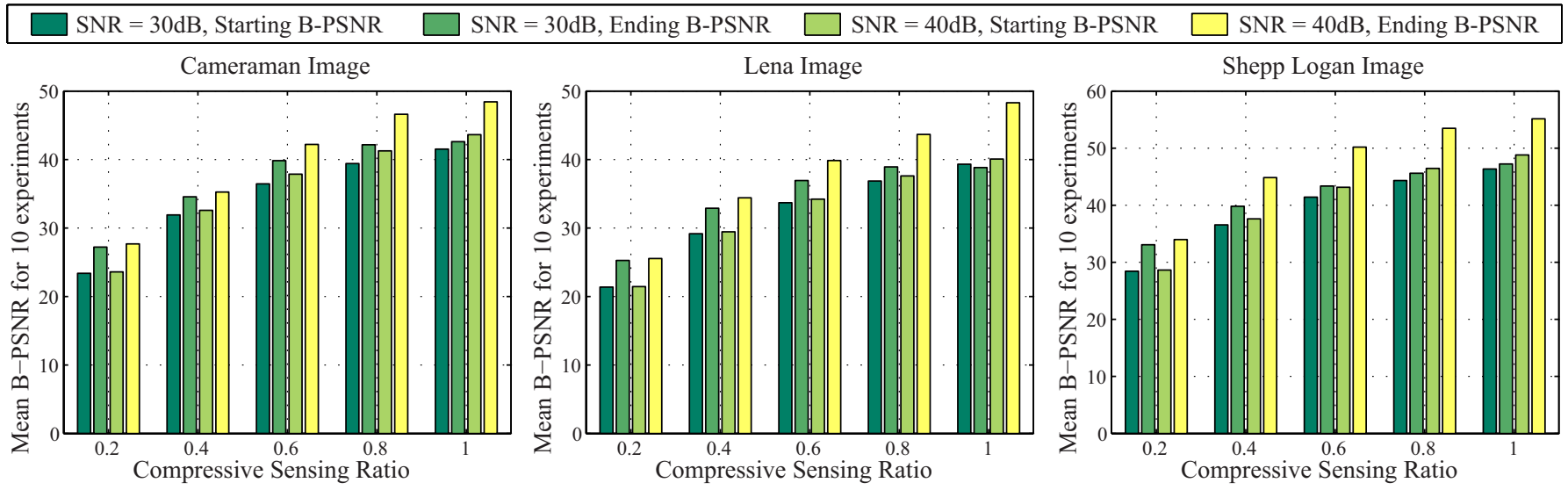

Fig. 9. Average starting (B-PSNR $\hat{\mathbf{z}}^{1}$ ) and ending (B-PSNR $\hat{\mathbf{z}}_{f}$ ) Blurred PSNRs, using the proposed CS BID algorithm, for all tested images. All images are degraded with a Gaussian PSF of variance 5 and Gaussian noise is added to the $\mathrm{CS}$ measurements so that $\mathrm{SNR}=30 \mathrm{~dB}$ or $\mathrm{SNR}=40 \mathrm{~dB}$.

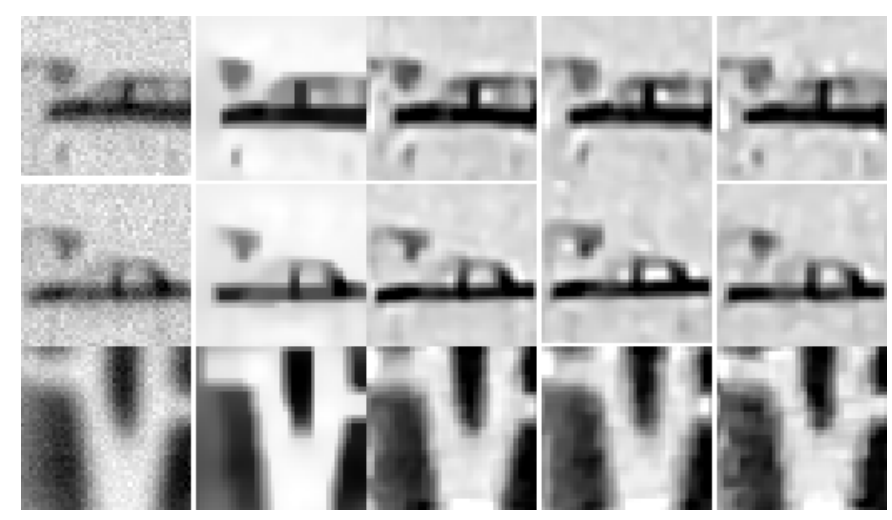

Fig. 10. Example restorations: $1^{\text {st }}$ column represents three different uncompressed blurred PMMW images of size $41 \times 43$ pixels, obtained from [31]. The PMMW images depict two cars and the middle part of a pair of scissors; $2^{\text {nd }}$ column represents the blind deconvolution result, without CS, using the algorithm in [34]; $3^{\text {rd }}, 4^{\text {th }}$, and $5^{\text {th }}$ columns represent, respectively, the restorations obtained by the proposed algorithm for compressive ratios $0.8,0.6$ and 0.4 , where $\Phi$ is a binary S-cyclic matrix (see [53]-[55] for more details).

sequential solution of problems (10) and (11). Let us denote the initially estimated blurred signal, resulting from the solution of (10), by $\hat{\mathbf{z}}^{1}=\mathbf{W a}^{1}$ and the final estimated blurred signal by $\hat{\mathbf{z}}^{k_{f}}=\mathbf{W} \mathbf{a}^{k_{f}}$, where $k_{f}$ denotes the final iteration $k$ of our proposed algorithm. We compare $\hat{\mathbf{z}}^{1}$ and $\hat{\mathbf{z}}^{k_{f}}$ to the original blurred signal $\mathbf{z}=\mathbf{H x}$ using the Blurred-PSNR (B-PSNR) metric denoted by

$$
\operatorname{B-PSNR}_{\hat{\mathbf{z}}}=10 \log _{10} \frac{N L^{2}}{\|\mathbf{z}-\hat{\mathbf{z}}\|^{2}},
$$

where the constant $L$ represents the maximum possible intensity value in image $\mathbf{z}$. High B-PSNR for a signal $\hat{\mathbf{z}}$ is expected to increase the accuracy to the solution of (11). Therefore, showing that B-PSNR $\hat{\mathbf{z}}^{k_{f}}>\mathrm{B}-\mathrm{PSNR}_{\hat{\mathbf{z}}^{1}}$, means that the simultaneous approach is able to provide improved performance compared to the sequential one, in the experiments.

Figure 9 shows the average starting $\left(\mathrm{B}-\mathrm{PSNR}_{\hat{\mathbf{z}}^{1}}\right)$ and ending $\left(\mathrm{B}^{-P_{S N R}} \hat{\mathbf{\mathbf { z }}}_{f}\right)$ B-PSNRs for all tested images when they are blurred with a Gaussian PSF of variance 5 for different noise
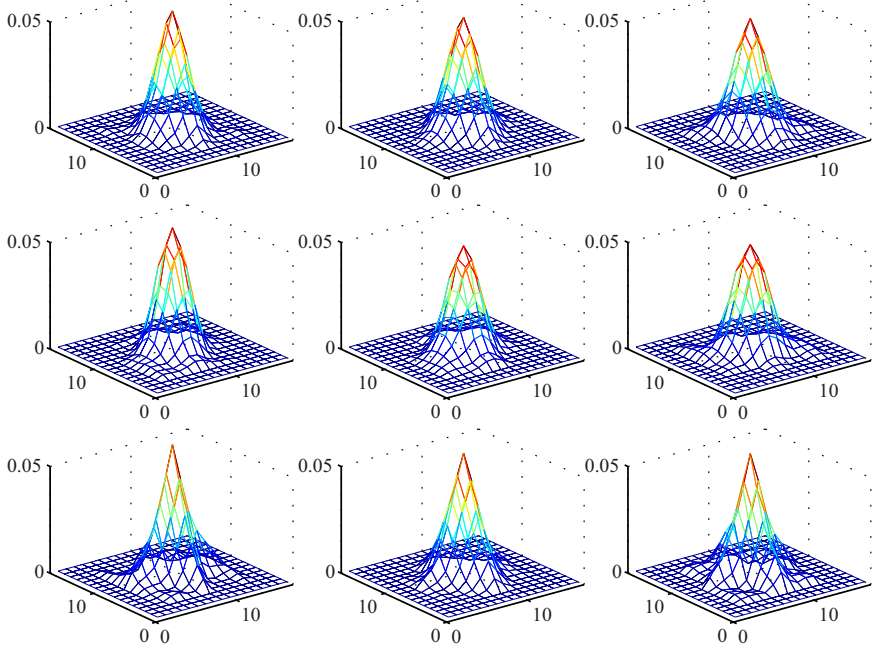

Fig. 11. Estimated PSFs $(17 \times 17$ central part $)$ for the blind reconstruction/restoration results of PMMW images, presented in the last three columns of Figure 10. $1^{\text {st }}$ and $2^{\text {nd }}$ rows correspond to the car images; $3^{\text {rd }}$ row corresponds to the scissors image for compressive ratios $0.8,0.6$ and 0.4 from left to right, respectively.

realizations. The clear increase in blurred signal quality at the final iteration of our algorithm effectively supports our previous discussion. Similar results, which are not reported here due to space limitations, were obtained for PSFs of different variance.

Let us emphasize that an appealing fact of our framework is that it is not restricted to specific algorithms when solving for the sparse vector a in equation (26). Therefore, if the accuracy of this step improves, higher image quality is expected to be obtained. In our implementation, we use the $l 1-l s$ algorithm, [21], for the solution of this step, but certainly the selection of another, more robust, algorithm could potentially provide improved performance overall.

In this work, we provide a robust framework, which combines and simultaneously solves a CS and BID problem. Unfortunately, we cannot utilize existing CS theory directly in our problem since the restricted isometry property (RIP) 
TABLE I

Average Performance Comparison of the Non-Blind and Blind Versions of the Proposed Approach With a Series of State-of-the-Art Algorithms, NAmely, ll-ls [21], GPSR [35], NESTA [36], YALL1 [37], and CoSaMP [38] That Solve the Non-Blind Reconstruction/Restoration Problem. EXPERIMENTS Were Conducted With Gaussian Blurs of DifFerent VARIANCE and VARIOUS

Noise Levels. For Each Tested Image (“CAmeraman", "LenA", "Shepp-Logan”) 10 Experiments Were Conducted for Each Compressive Ratio 0.2 to 1 With Step 0.2 and the Mean PNSR is Reported Here. Entries of the Table in Bold Denote the Highest AVERAge PSNR for EACH TeSted Set of: IMAge, Blur VARIAnCE, SNR And CS Ratio

\begin{tabular}{|c|c|c|c|c|c|c|c|c|c|c|c|c|c|c|c|c|c|c|c|}
\hline \multirow{3}{*}{\multicolumn{2}{|c|}{$\begin{array}{c}\text { Blur Var. } \\
\text { SNR }\end{array}$}} & \multicolumn{6}{|c|}{ Cameraman Image } & \multicolumn{6}{|c|}{ Lena Image } & \multicolumn{6}{|c|}{ Shepp-Logan Image } \\
\hline & & \multicolumn{2}{|c|}{3} & \multicolumn{2}{|c|}{-} & \multicolumn{2}{|c|}{9} & \multicolumn{2}{|c|}{3} & \multicolumn{2}{|c|}{5} & \multicolumn{2}{|c|}{9} & \multicolumn{2}{|c|}{3} & \multicolumn{2}{|c|}{ J } & \multicolumn{2}{|c|}{9} \\
\hline & & $30 \mathrm{~dB}$ & $40 \mathrm{~dB}$ & $30 \mathrm{~dB}$ & $40 \mathrm{~dB}$ & $30 \mathrm{~dB}$ & $40 \mathrm{~dB}$ & $30 \mathrm{~dB}$ & $40 \mathrm{~dB}$ & $30 \mathrm{~dB}$ & $40 \mathrm{~dB}$ & $30 \mathrm{~dB}$ & $40 \mathrm{~dB}$ & $30 \mathrm{~dB}$ & $40 \mathrm{~dB}$ & $30 \mathrm{~dB}$ & $40 \mathrm{~dB}$ & $30 \mathrm{~dB}$ & $40 \mathrm{~dB}$ \\
\hline Alg. & Ratic & \multicolumn{6}{|c|}{ Mean PSNR for 10 experiments } & \multicolumn{6}{|c|}{ Mean PSNR for 10 experiments } & \multicolumn{6}{|c|}{ Mean PSNR for 10 experiments } \\
\hline \multirow{5}{*}{$\frac{2}{1}$} & 0.2 & 21.46 & 23.75 & 20.72 & 22.69 & 19.98 & 21.35 & 23.13 & 26.54 & 22.30 & 25.09 & 22.35 & 23.53 & 21.61 & 22.78 & 21.17 & 22.02 & 20.19 & 20.46 \\
\hline & 0.4 & 22.29 & 24.18 & 21.12 & 23.02 & 20.48 & 21.54 & 24.11 & 27.26 & 23.04 & 25.58 & 22.50 & 23.73 & 21.75 & 23.10 & 21.45 & 22.27 & 20.42 & 20.72 \\
\hline & 0.6 & 22.68 & 24.44 & 21.35 & 23.17 & 20.66 & 21.66 & 24.63 & 27.59 & 23.36 & 25.86 & 22.78 & 23.90 & 21.89 & 23.29 & 21.58 & 22.32 & 20.52 & 20.81 \\
\hline & 0.8 & 22.90 & 24.55 & 21.51 & 23.30 & 20.75 & 21.72 & 24.98 & 27.82 & 23.53 & 26.02 & 22.87 & 23.96 & 22.01 & 23.47 & 21.59 & 22.35 & 20.61 & 20.85 \\
\hline & 1.0 & 23.03 & 24.70 & 21.65 & 23.39 & 20.82 & 21.82 & 25.21 & 27.90 & 23.67 & 26.15 & 23.01 & 24.09 & 22.11 & 23.65 & 21.69 & 22.40 & 20.65 & 20.90 \\
\hline \multirow{5}{*}{$\begin{array}{l}\frac{1}{5} \\
0\end{array}$} & 0.2 & 19.37 & 22.92 & 20.33 & 23.46 & 21.16 & 22.69 & 22.19 & 25.31 & 23.85 & 26.81 & 24.71 & 26.08 & 20.52 & 24.10 & 20.91 & 23.60 & 21.12 & 22.18 \\
\hline & 0.4 & 21.43 & 25.21 & 22.28 & 24.14 & 22.24 & 22.89 & 25.26 & 29.31 & 26.27 & 28.18 & 25.81 & 26.45 & 21.44 & 25.21 & 22.36 & 23.97 & 21.87 & 22.30 \\
\hline & 0.6 & 22.90 & 25.59 & 23.15 & 24.26 & 22.55 & 22.91 & 26.89 & 30.04 & 27.24 & 28.51 & 26.13 & 26.51 & 22.66 & 25.40 & 23.16 & 24.07 & 22.11 & 22.33 \\
\hline & 0.8 & 23.74 & 25.75 & 23.57 & 24.30 & 22.71 & 22.93 & 28.06 & 30.43 & 27.71 & 28.62 & 26.28 & 26.60 & 23.56 & 25.48 & 23.48 & 24.11 & 22.19 & 22.35 \\
\hline & 1.0 & 24.31 & 25.83 & 23.80 & 24.34 & 22.77 & 22.96 & 28.72 & 30.73 & 27.96 & 28.70 & 26.35 & 26.63 & 24.10 & 25.53 & 23.68 & 24.14 & 22.25 & 22.36 \\
\hline \multirow{5}{*}{$\begin{array}{l}\sum_{\tilde{n}}^{0} \\
0 \\
0 \\
0\end{array}$} & 0.2 & 14.84 & 21.69 & 14.60 & 22.17 & 15.07 & 21.83 & 16.44 & 23.89 & 16.12 & 24.91 & 16.88 & 25.06 & 17.54 & 22.88 & 17.57 & 22.56 & 18.28 & 21.89 \\
\hline & 0.4 & 15.27 & 23.45 & 16.18 & 23.28 & 17.22 & 22.75 & 16.76 & 26.20 & 17.99 & 26.69 & 19.54 & 26.36 & 19.25 & 23.80 & 19.30 & 23.28 & 19.80 & 22.21 \\
\hline & 0.6 & 16.47 & 24.31 & 17.58 & 23.89 & 18.68 & 23.06 & 18.01 & 27.41 & 19.52 & 27.62 & 21.03 & 26.85 & 20.61 & 24.19 & 20.41 & 23.53 & 20.68 & 22.36 \\
\hline & 0.8 & 17.39 & 24.85 & 18.52 & 24.26 & 19.45 & 23.29 & 19.14 & 28.18 & 20.60 & 28.08 & 21.90 & 27.08 & 21.44 & 24.55 & 21.20 & 23.61 & 21.18 & 22.38 \\
\hline & 1.0 & 18.37 & 25.29 & 19.54 & 24.50 & 20.01 & 23.40 & 20.05 & 28.70 & 21.67 & 28.56 & 22.94 & 27.19 & 21.94 & 24.55 & 21.68 & 23.63 & 21.43 & 22.41 \\
\hline \multirow{5}{*}{ 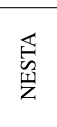 } & 0.2 & 22.19 & 23.80 & 21.80 & 22.93 & 21.04 & 21.48 & 24.83 & 26.50 & 24.27 & 25.38 & 23.20 & 23.64 & 22.37 & 22.84 & 21.72 & 22.08 & 20.44 & 20.54 \\
\hline & 0.4 & 22.48 & 24.25 & 22.08 & 23.27 & 21.45 & 21.86 & 25.28 & 27.27 & 24.70 & 25.91 & 23.67 & 24.05 & 22.78 & 23.19 & 22.02 & 22.26 & 20.81 & 20.84 \\
\hline & 0.6 & 22.76 & 24.48 & 22.35 & 23.43 & 21.74 & 22.10 & 25.65 & 27.59 & 25.05 & 26.19 & 23.95 & 24.22 & 23.09 & 23.43 & 22.22 & 22.39 & 21.05 & 21.00 \\
\hline & 0.8 & 22.92 & 24.60 & 22.52 & 23.53 & 21.78 & 22.21 & 25.86 & 27.76 & 25.30 & 26.39 & 23.98 & 24.38 & 23.22 & 23.54 & 22.26 & 22.47 & 21.17 & 21.15 \\
\hline & 1.0 & 22.88 & 24.63 & 22.35 & 23.44 & 21.72 & 22.11 & 25.75 & 27.76 & 24.99 & 26.20 & 23.99 & 24.28 & 23.25 & 23.64 & 22.24 & 22.42 & 21.15 & 20.95 \\
\hline & 0.2 & 22.62 & 22.81 & 21.41 & 21.49 & 20.69 & 20.77 & 24.61 & 24.75 & 23.38 & 23.46 & 22.30 & 22.38 & 22.02 & 22.07 & 20.69 & 20.71 & 19.42 & 19.44 \\
\hline$=$ & 0.4 & 23.13 & 23.29 & 21.83 & 21.94 & 20.88 & 20.93 & 25.48 & 25.66 & 23.85 & 23.93 & 22.70 & 22.76 & 22.30 & 22.33 & 20.97 & 20.99 & 19.61 & 19.62 \\
\hline $\bar{z}$ & 0.6 & 23.34 & 23.51 & 22.06 & 22.15 & 20.95 & 20.99 & 25.83 & 26.04 & 24.10 & 24.18 & 22.88 & 22.93 & 22.42 & 22.44 & 21.20 & 21.23 & 19.84 & 19.86 \\
\hline 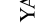 & 0.8 & 23.50 & 23.65 & 22.20 & 22.30 & 20.98 & 21.02 & 26.07 & 26.28 & 24.27 & 24.37 & 22.97 & 23.02 & 22.49 & 22.51 & 21.43 & 21.50 & 19.96 & 19.98 \\
\hline & 1.0 & 23.59 & 23.76 & 22.33 & 22.40 & 21.02 & 21.05 & 26.23 & 26.44 & 24.43 & 24.52 & 23.03 & 23.08 & 22.55 & 22.56 & 21.62 & 21.67 & 20.04 & 20.05 \\
\hline & 0.2 & 19.86 & 19.95 & 19.55 & 19.59 & 19.09 & 19.17 & 21.30 & 20.75 & 20.86 & 20.66 & 20.22 & 20.37 & 22.84 & 22.81 & 22.10 & 22.52 & 20.77 & 21.24 \\
\hline$\Xi \Xi$ & 0.4 & 24.58 & 25.05 & 23.68 & 24.09 & 22.67 & 22.99 & 26.98 & 27.79 & 26.00 & 26.97 & 24.80 & 25.77 & 24.99 & 25.81 & 23.87 & 24.74 & 22.21 & 23.10 \\
\hline & 0.6 & 25.44 & 26.10 & 24.28 & 24.76 & 23.10 & 23.47 & 28.74 & 30.55 & 27.35 & 28.88 & 25.83 & 27.10 & 25.47 & 26.36 & 24.27 & 25.18 & 22.58 & 23.56 \\
\hline & 0.8 & 25.68 & 26.42 & 24.46 & 24.98 & 23.25 & 23.63 & 29.48 & 31.41 & 27.94 & 29.52 & 26.30 & 27.55 & 25.67 & 26.63 & 24.44 & 25.40 & 22.80 & 23.80 \\
\hline & 1.0 & 25.83 & 26.59 & 24.57 & 25.09 & 23.34 & 23.74 & 29.89 & 31.83 & 28.29 & 29.85 & 26.60 & 27.82 & 25.79 & 26.79 & 24.54 & 25.53 & 22.91 & 23.93 \\
\hline & 0.2 & 19.05 & 19.10 & 19.01 & 19.06 & 18.46 & 18.53 & 21.07 & 19.37 & 20.68 & 19.62 & 19.40 & 19.28 & 22.77 & 21.48 & 22.07 & 21.25 & 20.21 & 19.42 \\
\hline & 0.4 & 22.07 & 22.16 & 21.88 & 21.75 & 21.65 & 21.46 & 26.00 & 25.25 & 25.19 & 25.31 & 23.88 & 24.64 & 24.83 & 25.71 & 23.76 & 24.67 & 21.85 & 22.33 \\
\hline & 0.6 & 21.83 & 21.02 & 23.50 & 23.72 & 22.93 & 23.25 & 27.25 & 26.53 & 26.18 & 27.70 & 24.59 & 26.06 & 25.11 & 26.15 & 23.98 & 25.02 & 22.15 & 22.48 \\
\hline 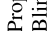 & 0.8 & 22.29 & 20.80 & 24.00 & 24.41 & 23.11 & 23.43 & 27.69 & 29.00 & 26.63 & 28.28 & 24.93 & 26.56 & 25.19 & 26.30 & 24.06 & 25.11 & 22.28 & 22.53 \\
\hline & 1.0 & 21.77 & 20.34 & 24.28 & 24.68 & 23.20 & 23.51 & 27.90 & 29.42 & 26.93 & 28.68 & 25.20 & 26.78 & 25.23 & 26.36 & 24.10 & 25.16 & 22.34 & 22.56 \\
\hline
\end{tabular}

might not always hold when the measurement matrix takes the form $\Phi \mathbf{H}$, and therefore restoration guarantees imposed by CS theory might not be achieved. However, as we have shown in section II, the blurred image $\mathbf{H x}$ is compressible and consequently can be recovered using existing CS techniques (in our case the measurement matrix is $\Phi$ alone), while the regularization terms on the image $\mathbf{x}$ and the blur $\mathbf{h}$ are employed to decouple the obtained signal Hx. In addition, the regularization term on the image (i.e., $R_{1}(\mathbf{x})$ ) aids in combating the ill-posedness of the BID sub-problem by appropriately modeling the sparse edges of the image (similar to TV regularization). Equivalent regularization terms have proven very efficient in modeling images in a series of recent BID algorithms presented in the literature, [40]-[47]. Furthermore, the introduced form of the constrained optimization problem is even more critical. The constraint $\mathbf{H x}=\mathbf{W a}$ which is incorporated in the minimization of equation (26) (step 3 of the algorithm) provides refinement in the estimation of $\mathbf{a}$ at each "outer-iteration" of the algorithm. Note that equation (26) not only tries to minimize the $\ell_{1}$ norm of the sparse coefficients a but, at the same time, tries to match them to the currently estimated blurred signal $\mathbf{H}^{k} \mathbf{x}^{k}$, hence providing increasingly accurate estimates per iteration. This behavior is also documented in the experimental results provided in Figure 9. Older methods, solving the minimization problems of equations (29) and (30), which clearly were not designed for the solution of the CS deblurring problem, lack this additional constraint. However, image deblurring is an illposed problem. Therefore, direct minimization of the $\ell_{1}$ norm of the sparse coefficients, without additional constraints to the solution, per iteration, is not able to provide as high performance, at least for CS ratios greater than $40 \%$, as the provided experimental results of Figure 5 and Table I document.

The average time needed to complete one run of the proposed algorithm for the tested images (size $256 \times 256$ pixels) is around $4-5$ minutes. The experiments were conducted using MATLAB on a $2.7 \mathrm{GHz}$ 4-core workstation with $4 \mathrm{~GB}$ of RAM. However, our implementation is not optimized for computational complexity purposes. The main goal of our work is to develop a general framework for CS BID problems where off-the-shelf CS algorithms can be selected for the solution of step 3 of the algorithm as well as appropriately chosen priors for the image and blur modeling. Therefore, computational complexity is directly tied to the chosen methods for the solution of each step. 


\section{B. PMMW Imaging and Experiments}

As mentioned in section I, the observation model in (2) can be beneficial in numerous applications, ranging from aerospace optical imaging devices to communications. Another important area where CS ideas have been recently incorporated, for cost reduction, is imaging systems for low-energy radiation spectra (e.g., PMMW imaging). PMMW imagers collect natural millimeter-wave radiation from the scene. The literature on PMMW imaging technology is rich (see [48] for a review). The millimeter-wave regime is part of the electromagnetic spectrum, in the frequency band between 30 and $300 \mathrm{GHz}$, and has significant advantages over conventional (e.g., visible light) imaging in low-visibility conditions during day or night. In environments dominated by clouds, fog, smoke, rain, snow and dust-storms, the millimeter-wave radiation is attenuated a few orders of magnitude less than the visual or infrared radiation. The ability to capture the scene in low-visibility conditions has led to various applications of PMMW technology over the course of years, [48]. Furthermore, advances in millimeter-wave radiometry (e.g., [49]) and integrated-circuit technologies (e.g., [50]) extended their use while technological breakthroughs led to the development of PMMW video devices (e.g., [51]). More recently, active and passive millimeter-wave scanners have been successfully introduced at airports to detect a broad range of concealed threats (e.g., [52]).

Conventional PMMW imaging systems consist of two main components, [31]. The first is responsible for measuring the incoming radiation while the second is responsible for directing the measurements and forming the image. The main disadvantage of these early devices is their complexity and their long acquisition times. Recently, several imaging systems have been proposed, [31], [53]-[56], to overcome these problems by incorporating CS concepts.

We now evaluate the performance of the proposed algorithm using real PMMW images (from [31]). To emphasize on the practical applications of our approach, we test our algorithm using binary (0/1) measurement matrices which are realizable in the millimeter-wave spectrum. Specifically, we employ binary S-cyclic matrices for PMMW imaging systems, proposed and analyzed by the authors in [53]-[55]. For such matrices, the size of the image must be restricted to $p \times q$ where $p$ and $q$ are consecutive prime numbers. Hence, we use PMMW images of size $41 \times 43$ pixels to perform experiments. In this experiment, the parameter space was set to $\left\{\alpha, \beta, \gamma, \tau, \eta^{1}\right\}=\left\{204.8,76 e 3,8 e 5,100, \beta / 2^{7}\right\}$. Also, we assume a priori that the size of the blur is $11 \times 11$, and enforce symmetry on the blur estimate after each iteration.

The resulting images, using this realizable sampling scheme, are presented in Figure 10 for different compressive ratios. It is evident that the resulting images are effectively enhanced and the blur is removed to a high extent. Note that the blurred-uncompressed images on the $1^{\text {st }}$ column of Figure 10 correspond to the best achievable reconstruction when the blurring function is not taken into consideration. Furthermore, for comparison purposes, we provide a blind deconvolution result, without CS, using the method presented in [34].
Figure 11 depicts the estimated PSFs, using our approach, for the reconstruction/restoration of the PMMW images in Figure 10. One can observe the consistency of the PSF estimation across different compressive ratios for each image. Moreover, the estimated PSFs for the two car images (first two rows) look similar, while the PSF shape for the scissors image (third row) appears spikier. Both car images were obtained in an outdoors environment, whereas, the scissors image was acquired indoors. The different acquisition configuration (positioning of lenses, distance between object and imager) supports such variations to the PSF shape.

\section{CONCLUSION}

In this paper, we presented a novel blind image deconvolution (BID) framework for imaging systems based on the principles of compressive sensing (CS). The proposed algorithm solves a constrained optimization problem reformulating it into a series of unconstrained sub-problems using the quadratic penalty method. It extends existing CS state-of-theart algorithms that cannot be easily employed for the solution of the BID problem. In addition, the proposed framework is general and can be easily adapted to accomodate different BID approaches which utilize alternative regularization terms for the image and blur. Simultaneous reconstructions and restorations of blurred synthetic images and real PMMW images are presented to demonstrate the robustness of our approach. We clearly show that modeling of the unknown blur is desirable when restoring images obtained through lensbased CS imaging systems.

\section{ACKNOWLEDGMENT}

The authors would like to thank N. Gopalsami, T. W. Elmer, S. Liao, and A. C. Raptis from the Nuclear Engineering (NE) Division of the Argonne National Laboratory (ANL) for providing them with the PMMW image data used for their experiments in Section IV-B.

\section{REFERENCES}

[1] B. Amizic, L. Spinoulas, R. Molina, and A. K. Katsaggelos, "Compressive sampling with unknown blurring function: Application to passive millimeter-wave imaging," in Proc. IEEE Int. Conf. Image Process., Oct. 2012, pp. 925-928.

[2] M. F. Duarte, M. A. Davenport, D. Takhar, J. N. Laska, T. Sun K. F. Kelly, and R. G. Baraniuk, "Single-pixel imaging via compressive sampling," IEEE Signal Process. Mag., vol. 25, no. 2, pp. 83-91, Mar. 2008.

[3] R. Fergus, A. Torralba, and W. T. Freeman, "Random lens imaging," Dept. Comput. Sci. Artif. Intell. Lab, Massachusetts Inst. Technol., Cambridge, MA, USA, Tech. Rep. MIT CSAIL TR 2006-058, 2006.

[4] L. McMackin, M. A. Herman, B. Chatterjee, and M. Weldon, "A highresolution swir camera via compressed sensing," Proc. SPIE, vol. 8353, no. 1, p. 835303, 2012.

[5] E. Candès, J. Romberg, and T. Tao, "Robust uncertainty principles: Exact signal reconstruction from highly incomplete frequency information," IEEE Trans. Inf. Theory, vol. 52, no. 2, pp. 489-509, Feb. 2006.

[6] Y. Tsaig and D. Donoho, "Compressed sensing," IEEE Trans. Inf. Theory, vol. 52, no. 4, pp. 1289-1306, Apr. 2006.

[7] E. J. Candès and M. B. Wakin, "An Introduction to compressive sampling," IEEE Signal Process. Mag., vol. 25, no. 2, pp. 21-30, Mar. 2008.

[8] V. Cevher, M. F. Duarte, C. Hedge, and R. Baraniuk, "Sparse signal recovery using Markov random fields," in Proc. Neural Inf. Process. Syst., 2008, pp. 1-8. 
[9] C. Hegde and R. G. Baraniuk, "Compressive sensing of streams of pulses," in Proc. 47th Annu. Allerton Conf. Commun., Control, Comput., 2009, pp. 44-51.

[10] C. Hegde and R. G. Baraniuk, "Sampling and recovery of pulse streams," IEEE Trans. Signal Process., vol. 59, no. 4, pp. 1505-1517, Apr. 2011.

[11] D. L. Snyder, Random Point Processes. New York, NY, USA: Wiley, 1975.

[12] C. Andrieu, E. Barat, and A. Doucet, "Bayesian deconvolution of noisy filtered point processes," IEEE Trans. Signal Process., vol. 49, no. 1, pp. 134-146, Jan. 2001

[13] J. M. Mendel, Optimal Seismic Deconvolution: An Estimation Based Approach. San Diego, CA, USA: Academic Press, 1983.

[14] E. A. Robinson, "Seismic time-invariant convolutional model," Geophysics, vol. 50, no. 12, pp. 2742-2751, 1985.

[15] M. Zhao and V. Saligrama, "On compressed blind de-convolution of filtered sparse processes," in Proc. IEEE Int. Conf. Acoust. Speech Signal Process., Mar. 2010, pp. 4038-4041.

[16] J. Ma and F.-X. Le Dimet, "Deblurring from highly incomplete measurements for remote sensing," IEEE Trans. Geosci. Remote Sens., vol. 47, no. 3, pp. 792-802, Mar. 2009.

[17] L. Xiao, J. Shao, L. Huang, and Z. Wei, "Compounded regularization and fast algorithm for compressive sensing deconvolution," in Proc. 6th Int. Conf. Image Graph., 2011, pp. 616-621.

[18] T. Edeler, K. Ohliger, S. Hussmann, and A. Mertins, "Super-resolution model for a compressed-sensing measurement setup," IEEE Trans. Instrum. Meas., vol. 61, no. 5, pp. 1140-1148, May 2012.

[19] E. J. Candès and T. Tao, "Decoding by linear programming," IEEE Trans. Inf. Theory, vol. 51, no. 12, pp. 4203-4215, Dec. 2005.

[20] J. Langford, L. Li, and T. Zhang, "Sparse online learning via truncated gradient,” J. Mach. Learn. Res., vol. 10, pp. 777-801, Jun. 2009.

[21] S.-J. Kim, K. Koh, M. Lustig, S. Boyd, and D. Gorinevsky, "An interiorpoint method for large-scale 11-regularized least squares," IEEE J. Sel. Topics Signal Process., vol. 1, no. 4, pp. 606-617, Dec. 2007.

[22] S. D. Babacan, R. Molina, and A. K. Katsaggelos, "Bayesian compressive sensing using Laplace priors," IEEE Trans. Image Process., vol. 19 no. 1, pp. 53-64, Jan. 2010.

[23] E. J. Candès and J. Romberg, "Practical signal recovery from random projections," in Proc. Wavelet Appl. Signal Image Process. 6th, Conf., 2005, pp. 76-86.

[24] V. Cevher, "Learning with compressible priors," in Proc. Neural Inf. Process. Syst., 2009, pp. 261-269.

[25] T. E. Bishop, S. D. Babacan, B. Amizic, A. K. Katsaggelos, T. Chan, and R. Molina, Blind Image Deconvolution: Problem Formulation and Existing Approaches. Cleveland, OH, USA: CRC Press, 2007.

[26] L. I. Rudin, S. Osher, and E. Fatemi, "Nonlinear total variation based noise removal algorithms," in Proc. 11th Annu. Int. Conf. Center Nonlinear Stud. Experim. Math., Comput. Issues Nonlinear Sci., 1992, pp. 259-268.

[27] J. Nocedal and S. J. Wright, Numerical Optimization. New York, NY, USA: Springer-Verlag, 2006.

[28] D. Krishnan and R. Fergus, "Fast image deconvolution using hyperLaplacian priors," in Proc. Adv. Neural Inf. Process. Syst., 2009, pp. 1033-1041.

[29] A. Levin, R. Fergus, F. Durand, and W. T. Freeman, "Image and depth from a conventional camera with a coded aperture," ACM Trans. Graph. vol. 26, no. 3, pp. 1-8, Jul. 2007.

[30] R. Molina, J. Mateos, and A. Katsaggelos, "Blind deconvolution using a variational approach to parameter, image, and blur estimation," IEEE Trans. Image Process., vol. 15, no. 12, pp. 3715-3727, Dec. 2006.

[31] N. Gopalsami, S. Liao, T. Elmer, A. Heifetz, and A. Raptis, "Compressive sampling in active and passive millimeter-wave imaging," in Proc. 36th Int. Conf. Infr., Millim. Terahertz Waves, Oct. 2011, pp. 1-2.

[32] Y. Li, J. Archer, J. Tello, G. Rosolen, F. Ceccato, S. Hay, A. Hellicar, and Y. Guo, "Performance evaluation of a passive millimeter-wave imager," IEEE Trans. Microw. Theory Technol., vol. 57, no. 10, pp. 2391-2405, Oct. 2009.

[33] J. Bioucas-Dias, M. Figueiredo, and J. Oliveira, "Total-variation image deconvolution: A majorization-minimization approach," in Proc. IEEE Int. Conf. Acoust. Speech Signal Process., May 2006, pp. 1-2.
[34] B. Amizic, S. D. Babacan, R. Molina, and A. K. Katsaggelos, "Sparse bayesian blind image deconvolution with parameter estimation," in Proc. Eur. Signal Process. Conf., 2010, pp. 626-630.

[35] M. Figueiredo, R. Nowak, and S. Wright, "Gradient projection for sparse reconstruction: Application to compressed sensing and other inverse problems," IEEE J. Sel. Topics Signal Process., vol. 1, no. 4 pp. 586-597, Dec. 2007.

[36] S. Becker, J. Bobin, and E. J. Candés, "NESTA: A fast and accurate first-order method for sparse recovery," SIAM J. Imag. Sci., vol. 4, no. 1, pp. 1-39, 2011.

[37] J. Yang and Y. Zhang, "Alternating direction algorithms for $\ell$ 1-problems in compressive sensing," SIAM J. Sci. Comput., vol. 1, pp. 250-278, Jan. 2011.

[38] D. Needell and J. A. Tropp, "CoSaMP: Iterative signal recovery from incomplete and inaccurate samples," Commun. ACM, vol. 53, no. 12, pp. 93-100, 2010.

[39] W. Yin, S. Morgan, J. Yang, and Y. Zhang, "Practical compressive sensing with Toeplitz and circulant matrices," Proc. SPIE, vol. 7744, p. $77440 \mathrm{~K}$, Jul. 2010 .

[40] R. Fergus, B. Singh, A. Hertzmann, S. T. Roweis, and W. T. Freeman, "Removing camera shake from a single photograph," ACM Trans. Graph., vol. 25, no. 3, pp. 787-794, 2006.

[41] Q. Shan, J. Jia, and A. Agarwala, "High-quality motion deblurring from a single image," in Proc. SIGGRAPH ACM SIGGRAPH, 2008, pp. $1-10$.

[42] S. Cho and S. Lee, "Fast motion deblurring," ACM Trans. Graph. vol. 28, no. 5, pp. 1-8, 2009

[43] A. Levin, Y. Weiss, F. Durand, and W. Freeman, "Understanding and evaluating blind deconvolution algorithms," in Proc. IEEE Conf. Comput. Vis. Pattern Recognit., Jun. 2009, pp. 1964-1971.

[44] L. Xu and J. Jia, "Two-phase kernel estimation for robust motion deblurring," in Proc. Eur. Conf. Comput. Vis., Part I, 2010, pp. 157-170.

[45] A. Levin, Y. Weiss, F. Durand, and W. Freeman, "Efficient marginal likelihood optimization in blind deconvolution," in Proc. IEEE Conf. Comput. Vis. Pattern Recognit., Jun. 2011, pp. 2657-2664.

[46] D. Krishnan, T. Tay, and R. Fergus, "Blind deconvolution using a normalized sparsity measure," in Proc. IEEE Conf. Comput. Vis. Pattern Recognit., Jun. 2011, pp. 233-240.

[47] S. Babacan, R. Molina, M. Do, and A. Katsaggelos, "Blind deconvolution with general sparse image priors," in Proc. Eur. Conf. Comput. Vis., Sep. 2012, pp. 341-355.

[48] L. Yujiri, M. Shoucri, and P. Moffa, "Passive millimeter wave imaging," IEEE Microw. Mag., vol. 4, no. 3, pp. 39-50, Sep. 2003.

[49] R. H. Dicke, "The measurement of thermal radiation at microwave frequencies," Rev. Sci. Instrum., vol. 17, no. 7, pp. 268-275, 1946.

[50] H. P. Moyer, J. J. Lynch, J. N. Schulman, R. L. Bowen, J. H. Schaffner, A. K. Kurdoghlian, and T. Y. Hsu, "A low noise chipset for passive millimeter wave imaging," in Proc. IEEE Int. Microw. Symp., Jun. 2007, pp. 1363-1366.

[51] J. A. Lovberg, C. Martin, and V. Kolinko, "Video-rate passive millimeter-wave imaging using phased arrays," in Proc. IEEE Int. Microw. Symp., Jun. 2007, pp. 1689-1692.

[52] O. Martinez, L. Ferraz, X. Binefa, I. Gomez, and C. Dorronsoro, "Concealed object detection and segmentation over millimetric waves images," in Proc. IEEE Comput. Soc. Conf. Comput. Vis. Pattern Recognit. Workshops, May 2010, pp. 31-37.

[53] S. D. Babacan, M. Luessi, L. Spinoulas, A. K. Katsaggelos, N. Gopalsami, T. Elmer, R. Ahern, S. Liao, and A. Raptis, "Compressive passive millimeter-wave imaging," in Proc. IEEE Int. Conf. Image Process., Sep. 2011, pp. 2705-2708.

[54] N. Gopalsami, S. Liao, T. W. Elmer, E. R. Koehl, A. Heifetz A. C. Raptis, L. Spinoulas, and A. K. Katsaggelos, "Passive millimeter wave imaging with compressive sensing," Opt. Eng., vol. 51, pp. 0916141-1-0916141-9, Sep. 2012.

[55] L. Spinoulas, J. Qi, A. K. Katsaggelos, T. W. Elmer, N. Gopalsami, and A. C. Raptis, "Optimized compressive sampling for passive millimeter-wave imaging," Appl. Opt., vol. 51, no. 26, pp. 6335-6342, Sep. 2012.

[56] V. M. Patel and J. N. Mait, "Compressive passive millimeter wave imaging with extended depth of field," Opt. Eng., vol. 51, no. 9, p. $091610,2012$. 


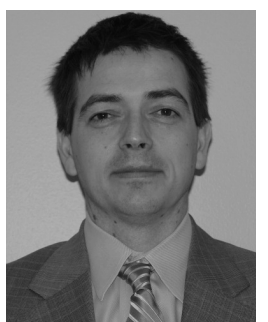

Bruno Amizic (M'11) was born in Split, Croatia. He received the B.S. and M.S. degrees in electrical engineering from the University of Missouri-Rolla, Rolla, MO, in 1999 and 2002, respectively. He is currently pursuing the Ph.D. degree in electrical engineering with Northwestern University, Evanston, IL, USA.

In 2002, he joined Zenith Electronics Corporation, Lincolnshire, IL, USA, where he worked on algorithms for improving the reception of terrestrial high definition television under channel disturbances like average white Gaussian noise, multipath, Doppler distortions, phase distortions due to tuner imperfections, and sampling frequency drifts between the transmitter and receivers sampling clocks. He joined Motorola, Schaumburg, IL, USA, in 2005, where he developed algorithms for the $3 \mathrm{G}$ mobile systems. In 2011, he joined The Boeing Company, Chicago, IL, USA.

Mr. Amizic holds four U.S. patents. His current research interests include Bayesian methods, compressive sensing, sparse statistical modeling, adaptive signal processing, deblurring, blind deconvolution, cybersecurity, and largescale (real-time) machine learning.

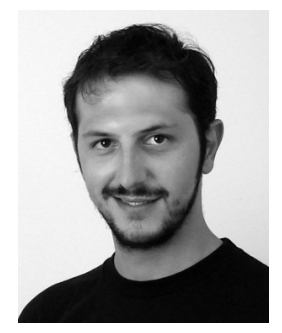

Leonidas Spinoulas (S'13) was born in Korinthos, Greece, in 1986. He received the B.S. degree in electrical and computer engineering from the National Technical University of Athens, Athens, Greece, in 2010, and the M.S. degree in electrical engineering and computer science from Northwestern University, Evanston, IL, USA, in 2012, where he is currently pursuing the $\mathrm{Ph} . \mathrm{D}$. degree in electrical engineering.

His current research interests include compressive sensing, sparse representations, signal and image restoration, deblurring, blind deconvolution, inverse problems, Bayesian methods, and computer vision.

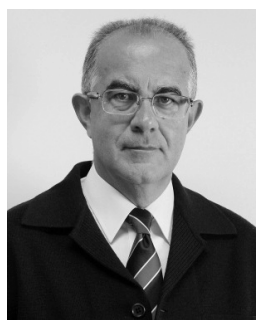

Rafael Molina (M'88) was born in 1957. He received the Degree in mathematics (statistics) in 1979 and the Ph.D. degree in optimal design in linear models in 1983. He became a Professor of computer science and artificial intelligence with the University of Granada, Granada, Spain, in 2000. He was a Former Dean with the Computer Engineering School, University of Granada, from 1992 to 2002 , and the Head of the Computer Science and Artificial Intelligence Department, University of Granada, from 2005 to 2007 .
His current research interests include using Bayesian modeling and inference in problems like image restoration (applications to astronomy and medicine), super resolution of images and video, blind deconvolution, computational photography, source recovery in medicine, compressive sensing, low rank matrix decomposition, active learning, and classification.

Dr. Molina serves the IEEE and other Professional Societies, include Applied Signal Processing, an Associate Editor of the IEEE TRANS ACTIONS ON IMAge Processing from 2005 to 2007, an Associate Editor of Progress in Artificial Intelligence in 2010, an Associate Editor of Digital SignAL PROCESSING in 2011, and an Area Editor in 2011. He is a recipient of the IEEE International Conference on Image Processing Paper Award in 2007, the ISPA Best Paper Award in 2009. He received the Runner-Up Prize at Reception for early-stage researchers at the House of Commons.

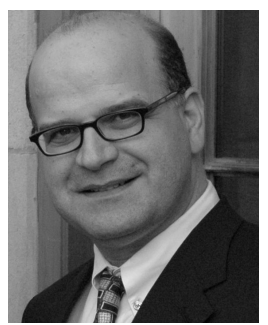

Aggelos K. Katsaggelos (F'98) received the Diploma degree in electrical and mechanical engineering from the Aristotelian University of Thessaloniki, Thessaloniki, Greece, in 1979, and the M.S. and Ph.D. degrees in electrical engineering from the Georgia Institute of Technology, Atlanta, GA, USA, in 1981 and 1985, respectively.

In 1985, he joined the Department of Electrical Engineering and Computer Science, Northwestern University, Evanston, IL, USA, where he is currently a Professor and an AT\&T Chair. He was the Ameritech Chair of information technology from 1997 to 2003. He is the Director of the Motorola Center for Seamless Communications, a member of an Academic Staff, NorthShore University Health System, Evanston, IL, USA, an Affiliated Faculty with the Department of Linguistics and he has an appointment with the Argonne National Laboratory, Lemont, IL, USA.

Dr. Katsaggelos has published extensively in the areas of multimedia signal processing and communications over 180 journal papers, 450 conference papers, and 40 book chapters. He holds 20 international patents. He is the co-author of Rate-Distortion Based Video Compression (Kluwer, 1997), SuperResolution for Images and Video (Claypool, 2007), and Joint Source-Channel Video Transmission (Claypool, 2007). He was an Editor-in-Chief of the IEEE Signal Processing MAGaZINE from 1997 to 2002, a BOG Member of the IEEE Signal Processing Society from 1999 to 2001, and a Publication Board Member of the IEEE Proceedings from 2003 to 2007. He is a fellow of SPIE in 2009. He is a recipient of the IEEE Third Millennium Medal in 2000, the IEEE Signal Processing Society Meritorious Service Award in 2001, the IEEE Signal Processing Society Technical Achievement Award in 2010, the IEEE Signal Processing Society Best Paper Award in 2001, the IEEE ICME Paper Award in 2006, the IEEE ICIP Paper Award in 2007, and the ISPA Paper Award in 2009. He was a Distinguished Lecturer of the IEEE Signal Processing Society from 2007 to 2008. 